\title{
Asymptotic regularity of trajectory attractor and trajectory statistical solution for the 3D globally modified Navier-Stokes equations*
}

\author{
Caidi Zhao ${ }^{a}{ }^{\dagger} \quad$ Tomás Caraballo ${ }^{b \ddagger}$ \\ ${ }^{a}$ Department of Mathematics, Wenzhou University, \\ Wenzhou, Zhejiang Province, 325035, P. R. China \\ ${ }^{b}$ Departmento de Ecuaciones Diferenciales y Análisis Numérico \\ Facultad de Matemáticas, Universidad de Sevilla, \\ c/ Tarfia s/n, 41012-Sevilla, Spain
}

October 14, 2018

\begin{abstract}
We first prove the existence and regularity of the trajectory attractor for a threedimensional system of globally modified Navier-Stokes equations. Then we use the natural translation semigroup and trajectory attractor to construct the trajectory statistical solutions in the trajectory space. In our construction the trajectory statistical solution is an invariant Borel probability measure, which is supported by the trajectory attractor and is invariant under the action of the translation semigroup. As a byproduct of the regularity of the trajectory attractor, we obtain the asymptotic regularity of the trajectory statistical solution in the sense that it is supported by a set in the trajectory space in which all weak solutions are in fact strong solutions.
\end{abstract}

Keywords: Globally modified Navier-Stokes equations; Trajectory attractor; Trajectory statistical solution; Invariant measure; Asymptotic regularity

MSC2010: 35B41, 34D35, 76F20

\footnotetext{
* Supported by NSF of China with No.11271290 and by NSF of Zhejiang Province with No.LY17A010011. Also supported by FEDER and the Spanish Ministerio de Economía y Competitividad project MTM2015-63723-P and the Consejería de Innovación, Ciencia y Empresa (Junta de Andalucía) under Proyecto de Excelencia P12-FQM-1492.

${ }^{\dagger}$ Corresponding author E-mail: zhaocaidi2013@163.com.

${ }^{\ddagger}$ E-mail: caraball@us.es
} 


\section{Introduction}

We study the following three-dimensional (3D) system of globally modified NavierStokes equations

$$
\begin{aligned}
& \frac{\partial u}{\partial t}-\nu \Delta u+F_{N}\left(\|u\|_{V}\right)(u \cdot \nabla) u+\nabla p=g(x, t) \quad \text { in } \quad(0,+\infty) \times \Omega, \\
& \nabla \cdot u=0 \\
& u=0 \quad \text { on } \quad(0,+\infty) \times \partial \Omega \\
& u(x, 0)=u_{0}(x), \quad x \in \Omega,
\end{aligned}
$$

where the unknown functions $u=u(x, t)=\left(u_{1}(x, t), u_{2}(x, t), u_{3}(x, t)\right)$ and $p=p(x, t)$ denote, respectively, the velocity field of the fluid and the kinematic pressure; $g(x, t)=$ $\left(g_{1}(x, t), g_{2}(x, t), g_{3}(x, t)\right)$ is the density of volume forces and $\nu>0$ is the viscosity coefficient. In (1.1), $\|u\|_{V}=\|\nabla u\|_{\left(L^{2}(\Omega)\right)^{3}}$, and the function $F_{N}(\cdot):(0,+\infty) \mapsto(0,1]$ is defined as

$$
F_{N}(r)=\min \{1, N / r\}, \quad r \in(0,+\infty),
$$

where $N>0$ is given. In addition, $x=\left(x_{1}, x_{2}, x_{3}\right) \in \Omega$ and $\Omega \subset \mathbb{R}^{3}$ is an open bounded subset and smooth enough such that the Poincaré inequality holds true.

The above globally modified Navier-Stokes equations were initiated in the paper [3]. The modifying factor $F_{N}\left(\|u\|_{V}\right)$ depends on the norm $\|u\|_{V}=\|\nabla u\|_{\left(L^{2}(\Omega)\right)^{3}}$, which in turn depends on $\nabla u$ over the whole domain $\Omega$ and not just at or near the point $x \in \Omega$ under consideration. Essentially, it prevents large gradients dominating the dynamics and leading to explosions ( $[3,4])$.

The globally modified Navier-Stokes equations are interesting themselves, but, more importantly, can be used to obtain useful information about the Navier-Stokes equations. We know that the Navier-Stokes equations are valid for accurate description of the motion of the fluid only in the case that $|\nabla u|$ is relatively small (see [18]). Obviously, if $\|\nabla u\|_{\left(L^{2}(\Omega)\right)^{3}} \leqslant N$, then equations (1.1)-(1.2) reduce to the incompressible Navier-Stokes equations. So the number $N$ is the threshold value for $\nabla u$. Also, the globally modified Navier-Stokes equations were used as an intermediate step by Kloeden and Valero in [16] to prove that the attainability set of the weak solutions of the 3D Navier-Stokes equations which satisfy an energy constraint is compact and connected set in the weak topology.

Nowadays, the globally modified Navier-Stokes equations have been widely studied by several researchers in some articles, including the existence and uniqueness of weak and strong solutions $[3,5,10,15,25,27]$; well-posedness and pullback attractor with infinite delays [23,24], invariant measures and statistical solutions $[4,17,22,37]$, etc.

The invariant measures and statistical solutions have proven to be very useful in the understanding of turbulence in the case of Navier-Stokes equations (see Foias et al. [12]). The main reason is that the measurements of several aspects of turbulent

flows are actually measurements of time-average quantities. Statistical solutions have 
been introduced as a rigorous mathematical object to formalize the notion of ensemble average in the conventional statistical theory of turbulence. There are two main notions of statistical solutions, one introduced by Foias and Prodi [11] (will be called Foias-Prodi statistical solution) and the other by Vishik and Furshikov [29] (will be called VishikFurshikov statistical solution). The Foias-Prodi statistical solution defined in [11] is a family of Borel measures parametrized by the time variable and defined on the phase space of the Navier-Stokes equations, representing the probability distribution of the velocity field of the flow at each time. While the Vishik-Furshikov statistical solutions given in [29] is a single Borel measure on the space of trajectories, representing the probability distribution of the space-time velocity field.

We recognize that the Foias-Prodi statistical solutions are associated to some invariant measures defined on the phase space (independent of time $t$ ) of the addressed system, while the Vishik-Furshikov statistical solutions are associated to some invariant measures defined on the trajectory space (dependent of time $t$ ) of solutions. The invariant measures for well-posed dissipative systems were studied in a series of references (see [6, 19-22, 26, 33]). For instance, Łukaszewicz, Real and Robinson [21] used the notion of Generalized Banach limit to construct the invariant measures for general continuous dynamical systems on metric spaces. Later, Chekroun and Glatt-Holtz [6] improved the results of [21] to construct invariant measures for a broad class of dissipative autonomous dynamical systems. Recently, Łukaszewicz and Robinson [22] extended the result of [6] to construct invariant measures for dissipative non-autonomous dynamical systems.

The construction of invariant measures in [6] depends heavily on the existence of global attractor of the continuous semigroup generated by the solution operators, and in [22] the construction depends heavily on the pullback attractor of the continuous process associated to the solutions operators. The original motivation of this article is to use the natural translation semigroup and trajectory attractor to construct invariant space-time measure and Vishik-Furshikov statistical solution for the globally modified Navier-Stokes equations in trajectory space.

The first main result in this article is the existence and regularity of the trajectory attractor for the globally modified Navier-Stokes equations (1.1)-(1.2). For the existence we will employ the theory of Vishik and Chepyzhov [8,31]. Consider the natural translation semigroup $\{S(t)\}_{t \geqslant 0}$ defined on the trajectory space $\mathcal{T}_{H}^{\mathrm{tr}}$ and on the regular trajectory space $\mathcal{T}_{V}^{\mathrm{tr}}$ (see notations in $\S 2$ ). We will first prove that $\{S(t)\}_{t \geqslant 0}$ possesses respectively a trajectory attractor $\mathcal{A}_{H}^{\mathrm{tr}}$ in $\mathcal{T}_{H}^{\mathrm{tr}}$ and $\mathcal{A}_{V}^{\mathrm{tr}}$ in $\mathcal{T}_{V}^{\mathrm{tr}}$, and then reveal the regularity and structure of the trajectory attractor by showing

$$
\mathcal{A}_{H}^{\mathrm{tr}}=\mathcal{A}_{V}^{\mathrm{tr}}=\Pi_{+} \mathcal{K}_{X}=\left\{\left.u(\cdot)\right|_{[0,+\infty)} \mid u \in \mathcal{K}_{X}\right\},
$$

where $\mathcal{K}_{X}$, which is called the weak kernel (with $X=H$ ) or strong kernel (with $X=V$ ) of equations (1.1)-(1.2), is the set of all bounded complete trajectories of equations (1.1)-(1.2):

$\mathcal{K}_{X}=\left\{u(t), t \in \mathbb{R} \mid u(t)\right.$ is a weak solution of $(1.1)-(1.2)$ and $\|u(t)\|_{X} \leqslant C_{u}$ for $\left.t \in \mathbb{R}\right\}$. 
Equality (1.5) implies the trajectory asymptotic smooth effect of the globally modified Navier-Stokes equations in the sense that all bounded weak solutions $u(t)$ are in fact strong solution when $t \in \mathbb{R}_{+}$.

The theory of trajectory attractor was studied systematically by Vishik and Chepyzhov in [8]. Now this theory has been applied to several concrete nonlinear partial differential equations, including the 3D Navier-Stokes equations [30,31], 2D and 3D non-Newtonian fluids [34,35], 3D dissipative Euler equations [9], the convective Brinkman-Forchheimer equations [36].

The second main result in this article is concerned with the existence of invariant Borel probability measure and trajectory statistical solution for the globally modified Navier-Stokes equations in the trajectory space. We want to remark that the approach of $[6,22]$ to construct invariant measure can not be applied directly here. But our ideas are originated from $[6,22]$. The main difference is that we consider the invariant measure on the trajectory space $\mathcal{A}_{H}^{\mathrm{tr}}$ (or $\mathcal{A}_{V}^{\mathrm{tr}}$ ) other than on the phase space $H$. It is worth mentioning that our approach for constructing invariant measure and trajectory statistical solution can be raised to an abstract level for a broad class of evolutionary equations displaying the property of global existence of weak solutions without a known result of global uniqueness, such as the $2 \mathrm{D}$ dissipative Euler equations [9], the 3D MHD equations $[7,14,32,38]$ and the $3 \mathrm{D}$ convective Brinkman-Forchheimer equations [36]. The fundamental hypotheses on the concrete evolutionary equations is that the trajectory space is a metrizable normal topological space and the natural translation semigroup possesses a compact trajectory attractor.

The paper is arranged as follows. In section 2, we first introduce some notations and operators, then recall some known results concerning the existence, uniqueness and regularity of the solutions. In section 3 , we prove the existence and regularity of the trajectory attractor. In section 4 , we use the trajectory attractor and translation semigroup to construct the invariant Borel probability measure and trajectory statistical solution. Finally, we summarize the article and provide a remark in section 5 .

\section{Preliminaries}

In this section, we first introduce some notations and operators. Then we present some preliminaries including the existence and uniqueness of solutions, and a compact embedding theorem which is essential in our applications.

In this paper we use the following notations:

$\mathbb{R}=(-\infty,+\infty), \mathbb{R}_{+}=[0,+\infty) ;$

$\mathbb{L}^{p}(\Omega)=\left(L^{p}(\Omega)\right)^{3}$-the 3D Lebesgue space with norm $\|\cdot\|_{\mathbb{L}^{p}(\Omega)} ;\|\cdot\|_{\mathbb{L}^{2}(\Omega)}=\|\cdot\|$;

$\mathbb{H}^{m}(\Omega)=$ the $3 \mathrm{D}$ Sobolev space $\left\{\phi \in \mathbb{L}^{2}(\Omega) \mid \nabla^{k} \phi \in \mathbb{L}^{2}(\Omega), k \leqslant m\right\}$ with norm $\|\cdot\|_{\mathbb{H}^{m}(\Omega)}$;

$\mathbb{H}_{0}^{1}(\Omega)=$ closure of $\left\{\phi \mid \phi \in\left(\mathcal{C}_{0}^{\infty}(\Omega)\right)^{3}\right\}$ in $\mathbb{H}^{1}(\Omega)$ with norm $\|\cdot\|_{\mathbb{H}^{1}(\Omega)}$;

$\mathcal{V}=\left\{\phi \in\left(\mathcal{C}_{0}^{\infty}(\Omega)\right)^{3} \mid \nabla \cdot \phi=0\right\}$;

$H=$ closure of $\mathcal{V}$ in $\mathbb{L}^{2}(\Omega)$ with norm $\|\cdot\| ; H^{\prime}=$ dual space of $H$;

$V=$ closure of $\mathcal{V}$ in $\mathbb{H}^{1}(\Omega)$ with norm $\|\cdot\|_{V}=\|\cdot\|_{\mathbb{H}^{1}(\Omega)}$; 
$V^{\prime}=$ dual space of $V$ with norm $\|\cdot\|_{V^{\prime}}$;

$(\cdot, \cdot)$ - the inner product in $H,\langle\cdot, \cdot\rangle$ - the dual pairing between $V$ and $V^{\prime}$;

$\operatorname{dist}_{M}(X, Y)$ - the Hausdorff semidistance between $X \subseteq M$ and $Y \subseteq M$ defined by

$$
\operatorname{dist}_{M}(X, Y)=\sup _{x \in X} \inf _{y \in Y}\|x-y\|_{M} .
$$

" $\longrightarrow$ " and " $\longrightarrow$ " denote the strong and weak convergence, respectively.

To write problem (1.1)-(1.4) as a functional form, we next introduce some operators. Firstly, we consider the operator $A: V \mapsto V^{\prime}$ defined as

$$
\langle A u, v\rangle=(\nabla u, \nabla v), \quad u, v \in V .
$$

Denoting $D(A)=\mathbb{H}^{2}(\Omega) \cap V$, then $A u=-P \Delta u, \forall u \in D(A)$, is the Stokes operator, where $P$ is the Leray-Helmholtz projection from $\mathbb{L}^{2}(\Omega)$ onto $H$. Secondly, we define a continuous trilinear form

$$
b(u, v, w)=\sum_{i, j=1}^{3} \int_{\Omega} u_{i} \frac{\partial v_{j}}{\partial x_{i}} w_{j} \mathrm{~d} x, u, v, w \in \mathbb{H}_{0}^{1}(\Omega) .
$$

Note that $V \subseteq \mathbb{H}_{0}^{1}(\Omega)$ is a closed subspace, $b(u, v, w)$ is continuous on $V \times V \times V$, and

$$
b(u, v, w)=-b(u, w, v), b(u, v, v)=0, \forall u, v, w \in V .
$$

For any $u, v \in V$,

$$
\langle B(u, v), w\rangle=b(u, v, w), \quad \forall w \in V,
$$

defines a continuous function $B(u, v)$ on $V \times V$. We further set

$$
\begin{aligned}
b_{N}(u, v, w) & =F_{N}\left(\|v\|_{V}\right) b(u, v, w), \quad \forall u, v, w \in V . \\
\left\langle B_{N}(u, v), w\right\rangle & =b_{N}(u, v, w), \quad \forall u, v, w \in V .
\end{aligned}
$$

Note that the form $b_{N}(u, v, w)$ is linear in $u$ and $w$, but it is nonlinear in $v$. Obviously,

$$
b_{N}(u, v, v)=0, \forall u, v \in V .
$$

For above introduced operators, we select the following estimations.

Lemma 2.1. ( $[4,17])$ There exist two positive constants $c_{1}$ and $c_{2}$ depending only on $\Omega$ such that

$$
\begin{aligned}
& \left|b_{N}(u, v, w)\right| \leqslant c_{1} N\|u\|_{V}\|w\|_{V}, \quad \forall u, v, w \in V, \\
& \left|b_{N}(u, v, w)\right| \leqslant c_{2} N\|A u\|\|w\|, \quad \forall u \in D(A), v \in V, w \in H .
\end{aligned}
$$

With the above notations, we can express, excluding the pressure $p$, the functional form of problem (1.1)-(1.4) in the solenoidal vector field as following (see e.g. [4,17]):

$$
\begin{aligned}
& u^{\prime}(t)+\nu A u(t)+B_{N}(u(t), u(t))=g(t) \text { in } \mathcal{D}^{\prime}\left(0,+\infty ; V^{\prime}\right), \\
& u(x, 0)=u_{0}, \quad x \in \Omega,
\end{aligned}
$$

where $u^{\prime}(t)=\frac{\partial u(t)}{\partial t}$.

We next specify the definition of solutions to problem (2.4)-(2.5). 
Definition 2.1. Let $u_{0} \in H$ and $g \in L^{2}(0, T ; H)$ for any $T>0$. A weak solution of problem (2.4)-(2.5) is any function $u(x, t) \in L^{\infty}(0, T ; H) \cap L^{2}(0, T ; V)$ for all $T>0$, with $\left.u(x, t)\right|_{t=0}=u_{0}$, such that

$$
\frac{\mathrm{d}}{\mathrm{d} t}(u(t), \phi)+\nu\langle A u(t), \phi\rangle+\left\langle B_{N}(u(t), u(t)), \phi\right\rangle=(g(t), \phi), \quad \forall \phi \in V,
$$

holds in the distribution sense of $\mathcal{D}^{\prime}(0,+\infty)$. If $u(x, t)$ is a weak solution and furthermore $u(x, t) \in L^{\infty}(0, T ; V) \cap L^{2}(0, T ; D(A))$ for all $T>0$, then $u(x, t)$ is called a strong solution of problem (2.4)-(2.5).

For the existence and uniqueness of solutions to problem (2.4)-(2.5), we have the following result. We want to remark that the result of Lemma 2.2 (1) can be found in [27, Theorem 1.1] and [3, Theorem 7(b), (c)], and the result of Lemma $2.2(2)$ is from $[3$, Theorem $7(\mathrm{a})]$.

Lemma 2.2. ( $[3,27])$ Suppose that $g(t) \in L^{2}(0, T ; H)$ for all $T>0$ and let $u_{0} \in H$ be given. Then

(1) If $u_{0} \notin V$, there exists a unique weak solution $u$ of problem (2.4)-(2.5) satisfying

$$
u \in \mathcal{C}([\varepsilon, T] ; V) \cap L^{2}(\varepsilon, T ; D(A)), \quad \text { for all } \quad T>\varepsilon>0, \forall \varepsilon>0 .
$$

(2) If $u_{0} \in V$, there exists a unique strong solution $u$ of problem (2.4)-(2.5) satisfying

$$
u \in \mathcal{C}([0, T] ; V) \cap L^{2}(0, T ; D(A)), \text { for all } T>0 .
$$

We conclude this section with the following useful results.

Lemma 2.3. ( [8]) Let $Y$ be a Banach space and $E \hookrightarrow E_{0} \subseteq Y$. Also let the embedding $E \hookrightarrow E_{0}$ be compact. Set

$$
W_{\infty, p}(0, T ; E, Y)=\left\{\phi(t), t \in[0, T] \mid \phi(t) \in L^{\infty}(0, T ; E), \quad \phi^{\prime}(t) \in L^{p}(0, T ; Y)\right\},
$$

with the norm $\|\phi\|_{W_{\infty, p}}=\operatorname{esssup}_{t \in[0, T]}\left\{\|\phi(t)\|_{E}\right\}+\left(\int_{0}^{T}\left\|\phi^{\prime}\right\|_{Y}^{p}\right)^{\frac{1}{p}}$, where $p>1$. Then $W_{\infty, p}(0, T ; E, Y) \hookrightarrow \mathcal{C}\left([0, T] ; E_{0}\right)$ with compact embedding.

Lemma 2.4. ( [8]) Let $y(s), K(s) \in L_{l o c}^{1}(0,+\infty)$ and let

$$
-\int_{0}^{+\infty} y(s) \phi^{\prime}(s) \mathrm{d} s+\beta \int_{0}^{+\infty} y(s) \phi(s) \mathrm{d} s \leqslant \int_{0}^{+\infty} K(s) \phi(s) \mathrm{d} s
$$

holds for any $\phi(s) \in \mathcal{C}_{0}^{\infty}\left(\mathbb{R}_{+}\right), \phi(s) \geqslant 0$, where $\beta \in \mathbb{R}$. Then for any $t, \tau \in \mathbb{R}_{+}, t \geqslant \tau$ there holds

$$
y(t) e^{\beta t}-y(\tau)^{\beta \tau} \leqslant \int_{\tau}^{t} K(s) e^{\beta s} \mathrm{~d} s .
$$




\section{Existence and regularity of trajectory attractor}

In this section, we will introduce the definitions of trajectory space $\mathcal{T}_{H}^{\mathrm{tr}}$ and regular trajectory space $\mathcal{T}_{V}^{\mathrm{tr}}$ for equation (2.4), as well as the definitions of trajectory attractor $\mathcal{A}_{H}^{\mathrm{tr}}$ in $\mathcal{T}_{H}^{\mathrm{tr}}$ and $\mathcal{A}_{V}^{\mathrm{tr}}$ in $\mathcal{T}_{V}^{\mathrm{tr}}$, respectively. The main result of this section is the existence and regularity of trajectory attractor.

We set $H^{\eta}=(-\Delta)^{-\eta / 2} H$ in this paper, where $\eta>0$ and the Laplace operator $\Delta$ is taken with zero boundary condition $\left.u\right|_{\partial \Omega}=0$. We use $H^{-\eta}$ to denote the dual space of $H^{\eta}$. In the whole article, we take $0<\eta<1$ and thus the embeddings $H \hookrightarrow H^{-\eta}$ and $V \hookrightarrow H^{1-\eta}$ are compact. Therefore, we can use the compact embedding result in Lemma 2.3. In addition, we use $\Pi_{+}$to denote the restriction operator (with respect to time variable) to the semi-infinite interval $\mathbb{R}_{+}$. Analogously, $\Pi_{T}$ stands for the restriction operator to the interval $[0, T]$. For example, if $u(\cdot) \in \mathcal{C}\left(\mathbb{R}_{+} ; H^{-\eta}\right) \cap$ $L^{\infty}\left(\mathbb{R}_{+} ; H\right)$, then $\Pi_{T} u(\cdot) \in \mathcal{C}\left([0, T] ; H^{-\eta}\right) \cap L^{\infty}(0, T ; H) ; \Pi_{T} u(t)=u(t)$ if $t \in[0, T]$.

\section{Definition 3.1.}

(1) (Trajectory space) The trajectory space $\mathcal{T}_{H}^{+}$of equation (2.4) consists of functions $u \in L^{\infty}\left(\mathbb{R}_{+} ; H\right) \cap L_{\text {loc }}^{2}\left(\mathbb{R}_{+} ; V\right)$ such that for any $T>0$ the function $\Pi_{T} u(t)$ is a weak solution of $(2.4)$ on $[0, T]$.

(2) (Regular trajectory space) The regular trajectory space $\mathcal{T}_{V}^{+}$of equation (2.4) consists of functions $u \in L^{\infty}\left(\mathbb{R}_{+} ; V\right) \cap L_{\text {loc }}^{2}\left(\mathbb{R}_{+} ; D(A)\right)$ such that for any $T>0$ the function $\Pi_{T} u(t)$ is a strong solution of $(2.4)$ on $[0, T]$.

In the sequel, we let $\mathcal{C}\left([a, b] ; H^{-\eta}\right)$ be the space of continuous $H^{-\eta}$-valued functions on $[a, b]$ endowed with the metric

$$
\mathrm{d}_{\mathcal{C}\left([a, b] ; H^{-\eta}\right)}(u, v)=\sup _{t \in[a, b]} \mathrm{d}_{H^{-\eta}}(u(t), v(t)) .
$$

Also we denote by $\mathcal{C}\left([a,+\infty) ; H^{-\eta}\right)$ the space of continuous $H^{-\eta}$-valued functions on $[a,+\infty)$ endowed with the metric

$$
\mathrm{d}_{\mathcal{C}\left([a,+\infty) ; H^{-\eta}\right)}(u, v)=\sum_{T \in \mathbb{N}} \frac{1}{2^{T}} \frac{\sup \left\{\mathrm{d}_{H^{-\eta}}(u(t), v(t)): a \leqslant t \leqslant a+T\right\}}{1+\sup \left\{\mathrm{d}_{H^{-\eta}}(w(t), \phi(t)): a \leqslant t \leqslant a+T\right\}} .
$$

We will adopt the topology of $\mathcal{C}_{\text {loc }}\left(\mathbb{R}_{+} ; H^{-\eta}\right)$ as the topology of the trajectory space $\mathcal{T}_{H}^{\mathrm{tr}}$, where $\mathcal{C}_{\text {loc }}\left(\mathbb{R}_{+} ; H^{-\eta}\right)$ is endowed with the compact open topology. Endowed with this topology, the trajectory space $\mathcal{T}_{H}^{\mathrm{tr}}$ is metrizable. Similarly, the regular trajectory space $\mathcal{T}_{V}^{\mathrm{tr}}$ is also metrizable with the following metric

$$
\mathrm{d}_{\mathcal{C}\left([0,+\infty) ; H^{1-\eta}\right)}(u, v)=\sum_{T \in \mathbb{N}} \frac{1}{2^{T}} \frac{\sup \left\{\mathrm{d}_{H^{1-\eta}}(u(t), v(t)): 0 \leqslant t \leqslant T\right\}}{1+\sup \left\{\mathrm{d}_{H^{1-\eta}}(w(t), \phi(t)): 0 \leqslant t \leqslant T\right\}} .
$$

In addition, the natural translation semigroup $\{S(t)\}_{t \geqslant 0}$ on $\mathcal{T}_{H}^{\mathrm{tr}}$ and $\mathcal{T}_{V}^{\mathrm{tr}}$ is defined as

$$
S(t) u(\cdot)=u(t+\cdot), \forall u \in \mathcal{T}_{H}^{\mathrm{tr}} \text { or } \mathcal{T}_{V}^{\mathrm{tr}} .
$$

We next specify the definition of trajectory attractor. 


\section{Definition 3.2.}

(1) A set $\mathcal{A}_{H}^{\mathrm{tr}} \subseteq \mathcal{T}_{H}^{+}$is called the trajectory attractor of equation (2.4) with respect to the topology $\mathcal{C}_{\text {loc }}\left(\mathbb{R}_{+} ; H^{-\eta}\right)$ if

(i) $\mathcal{A}_{H}^{\mathrm{tr}}$ is compact in $\mathcal{C}_{\text {loc }}\left(\mathbb{R}_{+} ; H^{-\eta}\right)$ and bounded in $L^{\infty}\left(\mathbb{R}_{+} ; H\right)$;

(ii) $S(t) \mathcal{A}_{H}^{\mathrm{tr}}=\mathcal{A}_{H}^{\mathrm{tr}}, \forall t \geqslant 0$;

(iii) For any bounded (in $L^{\infty}\left(\mathbb{R}_{+} ; H\right)$ norm) set $\mathcal{B}_{H} \subset \mathcal{T}_{H}^{+}$and $\forall T \geqslant 0$,

$$
\lim _{t \rightarrow+\infty} \operatorname{dist}_{\mathcal{C}\left([0, T] ; H^{-\eta}\right)}\left(\Pi_{T} S(t) \mathcal{B}_{H}, \Pi_{T} \mathcal{A}_{H}^{\mathrm{tr}}\right)=0 .
$$

(2) A set $\mathcal{A}_{V}^{\mathrm{tr}} \subseteq \mathcal{T}_{V}^{+}$is called the trajectory attractor of equation (2.4) with respect to the topology $\mathcal{C}_{\text {loc }}\left(\mathbb{R}_{+} ; H^{1-\eta}\right)$ if

(i) $\mathcal{A}_{V}^{\text {tr }}$ is compact in $\mathcal{C}_{\text {loc }}\left(\mathbb{R}_{+} ; H^{1-\eta}\right)$ and bounded in $L^{\infty}\left(\mathbb{R}_{+} ; V\right)$;

(ii) $S(t) \mathcal{A}_{V}^{\mathrm{tr}}=\mathcal{A}_{V}^{\mathrm{tr}}, \forall t \geqslant 0$;

(iii) For any bounded (in $L^{\infty}\left(\mathbb{R}_{+} ; V\right)$ norm) set $\mathcal{B}_{V} \subset \mathcal{T}_{V}^{+}$and $\forall T \geqslant 0$,

$$
\lim _{t \rightarrow+\infty} \operatorname{dist}_{\mathcal{C}\left([0, T] ; H^{1-\eta}\right)}\left(\Pi_{T} S(t) \mathcal{B}_{V}, \Pi_{T} \mathcal{A}_{V}^{\mathrm{tr}}\right)=0
$$

We remark that a set $\mathcal{A}_{H}^{\text {tr }}$ satisfying property (1)(iii) of Definition 3.2 is called a trajectory attracting set of $\{S(t)\}_{t \geqslant 0}$ in $\mathcal{T}_{H}^{+}$. Similarly, a set $\mathcal{A}_{V}^{\text {tr }}$ satisfying property (2)(iii) of Definition 3.2 is called a trajectory attracting set of $\{S(t)\}_{t \geqslant 0}$ in $\mathcal{T}_{V}^{+}$. To prove the existence of the trajectory attractor in $\mathcal{T}_{H}^{+}$and $\mathcal{T}_{V}^{+}$respectively, we shall establish, according to the theory of Vishik and Chepyzhov [8], that $\mathcal{T}_{H}^{+}$and $\mathcal{T}_{V}^{+}$are translation invariant under the action of $\{S(t)\}_{t \geqslant 0}$, and $\{S(t)\}_{t \geqslant 0}$ possesses a compact attracting set in $\mathcal{T}_{H}^{+}$and $\mathcal{T}_{V}^{+}$, respectively.

From Lemma 2.2 and Definitions 3.2, we are ready to establish

Lemma 3.1. Let $g \in L^{2}(0, T ; H)$ for any $T>0$.

(1) (i) For any $u_{0} \in H$, there exists a trajectory $u(t) \in \mathcal{T}_{H}^{+}$such that $u(0)=u_{0}$;

(ii) $\mathcal{T}_{H}^{+}$is translation invariant under the action of $\{S(t)\}_{t \geqslant 0}$, i.e.,

$$
S(t) \mathcal{T}_{H}^{+} \subseteq \mathcal{T}_{H}^{+}, \quad \forall t \geqslant 0
$$

(2) (i) For any $u_{0} \in V$, there exists a trajectory $u(t) \in \mathcal{T}_{V}^{+}$such that $u(0)=u_{0}$;

(ii) $\mathcal{T}_{V}^{+}$is translation invariant under the action of $\{S(t)\}_{t \geqslant 0}$, i.e.,

$$
S(t) \mathcal{T}_{V}^{+} \subseteq \mathcal{T}_{V}^{+}, \quad \forall t \geqslant 0
$$

Further, we have the following inclusion relations. This is the reason that we endow $\mathcal{T}_{H}^{+}$with the topology of $\mathcal{C}_{\text {loc }}\left(\mathbb{R}_{+} ; H^{-\eta}\right)$, and endow $\mathcal{T}_{V}^{+}$with the topology of $\mathcal{C}_{\text {loc }}\left(\mathbb{R}_{+} ; H^{1-\eta}\right)$. 
Lemma 3.2. Let $g \in L^{2}(0, T ; H)$ for any $T>0$.

(1) $\mathcal{T}_{H}^{+} \subseteq \mathcal{C}_{\text {loc }}\left(\mathbb{R}_{+} ; H^{-\eta}\right) \cap L^{\infty}\left(\mathbb{R}_{+} ; H\right)$.

(2) $\mathcal{T}_{V}^{+} \subseteq \mathcal{C}_{\text {loc }}\left(\mathbb{R}_{+} ; H^{1-\eta}\right) \cap L^{\infty}\left(\mathbb{R}_{+} ; V\right)$.

Proof. To prove item Lemma 3.2(1), we first verify that

$$
\begin{aligned}
& t \mapsto A u \in L^{2}\left(0, T ; V^{\prime}\right), \forall u \in L^{\infty}(0, T ; H) \cap L^{2}(0, T ; V), \forall T>0 ; \\
& t \mapsto B_{N}(u, u) \in L^{2}\left(0, T ; V^{\prime}\right), \forall u \in L^{\infty}(0, T ; H) \cap L^{2}(0, T ; V), \forall T>0 .
\end{aligned}
$$

In fact, for any $v \in V$,

$$
|\langle A u, v\rangle|=|\langle\nabla u, \nabla v\rangle| \leqslant\|u\|_{V}\|v\|_{V}, \forall u \in V,
$$

thus

$$
\int_{0}^{T}\|A u(t)\|_{V^{\prime}}^{2} \mathrm{~d} t \leqslant\|u\|_{L^{2}(0, T ; V)}^{2}, \forall u \in L^{2}(0, T ; V),
$$

and (3.5) is proved. Also, by (2.2),

$$
\int_{0}^{T}\left\|B_{N}(u(t), u(t))\right\|_{V^{\prime}}^{2} \mathrm{~d} t \leqslant c_{1} N\|u\|_{L^{2}(0, T ; V)}^{2}
$$

and (3.6) is established. Since $g \in L^{2}(0, T ; H)$ for any $T>0$, we conclude from (3.5)(3.6) and equation (2.4) that $\partial_{t} u(\cdot) \in L_{l o c}^{2}\left(\mathbb{R}_{+} ; V^{\prime}\right)$. Since $H \hookrightarrow H^{-\eta} \subseteq V^{\prime}$ and the embedding $H \hookrightarrow H^{-\eta}$ is compact, we infer from Lemma 2.3 that $u(\cdot) \in \mathcal{C}_{\text {loc }}\left(\mathbb{R}_{+} ; H^{-\eta}\right)$. The inclusion relation in item Lemma 3.2(1) follows.

To prove item Lemma $3.2(2)$, we check that

$$
\begin{aligned}
& t \mapsto A u \in L^{2}(0, T ; H), \forall u \in L^{\infty}(0, T ; V) \cap L^{2}(0, T ; D(A)), \forall T>0 ; \\
& t \mapsto B_{N}(u, u) \in L^{2}(0, T ; H), \forall u \in L^{\infty}(0, T ; V) \cap L^{2}(0, T ; D(A)), \forall T>0 .
\end{aligned}
$$

Indeed, for any $u \in D(A)=\mathbb{H}^{2}(\Omega) \cap V$, we have $A u \in H$ and $\|A u\| \leqslant\|u\|_{D(A)}$. Thus

$$
|(A u, v)| \leqslant\|u\|_{D(A)}\|v\|, \forall v \in H,
$$

and, consequently,

$$
\int_{0}^{T}\|A u(t)\|^{2} \mathrm{~d} t \leqslant \int_{0}^{T}\|u(t)\|_{D(A)}^{2} \mathrm{~d} t=\|u\|_{L^{2}(0, T ; D(A))}^{2}, \forall u \in L^{2}(0, T ; D(A)) .
$$

We obtain (3.9). At the same time, (2.3) implies that

$$
\left\|B_{N}(u, u)\right\| \leqslant c_{2} N\|A u\| \leqslant c_{2} N\|u\|_{D(A)}, \forall u \in D(A),
$$

and thus

$$
\int_{0}^{T}\left\|B_{N}(u(t), u(t))\right\|^{2} \mathrm{~d} t \leqslant c_{2} N\|u\|_{L^{2}(0, T ; D(A))}^{2}
$$

We obtain (3.10). Note that $g \in L^{2}(0, T ; H)$ for any $T>0$, we infer from (3.9)(3.10) and equation (2.4) that $\partial_{t} u(\cdot) \in L_{\text {loc }}^{2}\left(\mathbb{R}_{+} ; H\right)$. Since $V \hookrightarrow H^{1-\eta} \subseteq H$ and the embedding $V \hookrightarrow H^{1-\eta}$ is compact, we infer also from Lemma 2.3 that $u(\cdot) \in$ $\mathcal{C}_{\text {loc }}\left(\mathbb{R}_{+} ; H^{1-\eta}\right)$. The proof of Lemma 3.2 is completed. 
We next estimate the solutions in $\mathcal{T}_{H}^{+}$and $\mathcal{T}_{V}^{+}$. Let $\lambda_{1}$ be the first eigenvalue of the Stokes operator $A$. Also, we will employ the notation $a \lesssim b$ to mean that $a \leqslant c b$ for a universal constant $c>0$ that only depends on the parameters coming from the problem.

Lemma 3.3. Suppose $g \in H$ and set $\gamma=\nu \lambda_{1}$.

(1) For any trajectory $u \in \mathcal{T}_{H}^{+}$, there exists a positive constant $R_{H}$ which is independent of $u$, such that

$$
\begin{aligned}
& \|S(t) u\|_{L^{\infty}\left(\mathbb{R}_{+} ; H\right)}+\|S(t) u\|_{L^{2}(0,1 ; V)}+\left\|S(t) \partial_{s} u\right\|_{L^{2}\left(0,1 ; V^{\prime}\right)} \\
= & \operatorname{esssup}_{s \geqslant t}\|u(s)\|+\left(\int_{t}^{t+1}\|u(s)\|_{V^{2}}^{2} \mathrm{~d} s\right)^{\frac{1}{2}}+\left(\int_{t}^{t+1}\left\|\partial_{s} u(s)\right\|_{V^{\prime}}^{2} \mathrm{~d} s\right)^{\frac{1}{2}} \\
\lesssim & \|u\|_{L^{\infty}(0,1 ; H)} e^{-\frac{\gamma t}{2}}+R_{H}, \quad \forall t \geqslant 1 .
\end{aligned}
$$

(2) For any trajectory $u \in \mathcal{T}_{V}^{+}$, there exists a positive constant $R_{V}$ which is independent of $u$, such that

$$
\begin{aligned}
& \|S(t) u\|_{L^{\infty}\left(\mathbb{R}_{+} ; V\right)}+\|S(t) u\|_{L^{2}(0,1 ; D(A))}+\left\|S(t) \partial_{s} u\right\|_{L^{2}(0,1 ; H)} \\
= & \operatorname{esssup}_{s \geqslant t}\|u(s)\|_{V}+\left(\int_{t}^{t+1}\|u(s)\|_{D(A)}^{2} \mathrm{~d} s\right)^{\frac{1}{2}}+\left(\int_{t}^{t+1}\left\|\partial_{s} u(s)\right\|^{2} \mathrm{~d} s\right)^{\frac{1}{2}} \\
\lesssim & \|u\|_{L^{\infty}(0,1 ; V)}\left(1+t+t^{2}\right)^{1 / 2} e^{-\frac{\gamma t}{2}}+R_{V}, \quad \forall t \geqslant 1 .
\end{aligned}
$$

Proof. We first prove (3.13). Let $u \in \mathcal{T}_{H}^{+}$. Using $u$ to take dual pairing $\langle\cdot, \cdot\rangle$ with equation (2.4) and also using (2.1) yield the following energy equality

$$
\frac{1}{2} \frac{\mathrm{d}}{\mathrm{d} t}\|u(t)\|^{2}+\nu\|\nabla u(t)\|^{2}=(g, u(t)), \forall t>0 .
$$

By the Schwartz inequality and the following Poincaré inequality

$$
\lambda_{1}\|\phi\|^{2} \leqslant\|\nabla \phi\|^{2}, \forall \phi \in \mathbb{H}_{0}^{1}(\Omega),
$$

we deduce from (3.15) that $\frac{1}{2} \frac{\mathrm{d}}{\mathrm{d} t}\|u(t)\|^{2}+\nu\|\nabla u(t)\|^{2} \leqslant \frac{\|g\|^{2}}{2 \nu \lambda_{1}}+\frac{\nu \lambda_{1}}{2}\|u(t)\|^{2}$, that is

$$
\frac{\mathrm{d}}{\mathrm{d} t}\|u(t)\|^{2}+\nu\|\nabla u(t)\|^{2} \leqslant \frac{\|g\|^{2}}{\nu \lambda_{1}}, \forall t>0 .
$$

Note that the energy inequality (3.17) is interpreted in the following sense

$$
-\int_{0}^{T}\|u(t)\|^{2} \psi^{\prime}(t) \mathrm{d} t+\nu \int_{0}^{T}\|\nabla u(t)\|^{2} \psi(t) \mathrm{d} t \leqslant \int_{0}^{T} \frac{\|g\|^{2}}{\nu \lambda_{1}} \psi(t) \mathrm{d} t,
$$

for any $T>0$ and any $\psi \in \mathcal{C}_{0}^{\infty}([0, T])$. Set $\gamma=\nu \lambda_{1}$, then (3.17) and above inequality imply

$$
\begin{aligned}
& -\int_{0}^{+\infty}\|u(t)\|^{2} \psi^{\prime}(t) \mathrm{d} t+\gamma \int_{0}^{+\infty}\|u(t)\|^{2} \psi(t) \mathrm{d} t \\
\leqslant & \int_{0}^{+\infty}\left(\frac{\|g\|^{2}}{\gamma}-\nu\left(\left\|\nabla u(t)-\lambda_{1}\right\| u(t) \|^{2}\right) \psi(t) \mathrm{d} t, \forall \psi \in \mathcal{C}_{0}^{\infty}\left(\mathbb{R}_{+}\right) .\right.
\end{aligned}
$$


Now (3.16), (3.18) and Lemma 2.4 yield

$$
\|u(t)\|^{2} e^{\gamma t}-\|u(\tau)\|^{2} e^{\gamma \tau} \leqslant \frac{1}{\gamma} \int_{\tau}^{t}\|g\|^{2} e^{\gamma s} \mathrm{~d} s, \forall t \geqslant \tau, t, \tau \in \mathbb{R}_{+},
$$

which implies that

$$
\begin{aligned}
\|u(t)\| & \leqslant\|u(\tau)\| e^{\gamma(\tau-t) / 2}+\frac{\|g\|}{\gamma} \leqslant e^{\gamma / 2} \sup _{\tau \in(0,1)}\|u(\tau)\| e^{-\gamma t / 2}+\frac{\|g\|}{\gamma} \\
& \lesssim\|u\|_{L^{\infty}(0,1 ; H)} e^{-\gamma t / 2}+\|g\|, \forall t \geqslant 1 .
\end{aligned}
$$

Now, by (3.16) and (3.17),

$$
\begin{aligned}
\|S(t) u(s)\|_{L^{2}(0,1 ; V)}^{2} & =\int_{t}^{t+1}\|u(s)\|_{V}^{2} \mathrm{~d} s=\int_{t}^{t+1}\left(\|u(s)\|^{2}+\|\nabla u(s)\|^{2}\right) \mathrm{d} s \\
& \lesssim \int_{t}^{t+1}\|\nabla u(s)\|^{2} \mathrm{~d} s \lesssim\|u(t)\|^{2}+\|g\|^{2}
\end{aligned}
$$

which, together with (3.19) gives directly that

$$
\|S(t) u(s)\|_{L^{2}(0,1 ; V)} \lesssim\|u\|_{L^{\infty}(0,1 ; H)} e^{-\gamma t / 2}+\|g\| .
$$

It then follows from (2.4), (3.7), (3.8) and (3.20) that

$$
\begin{aligned}
& \left(\int_{t}^{t+1}\left\|\partial_{s} u(s)\right\|_{V^{\prime}}^{2} \mathrm{~d} s\right)^{1 / 2} \\
\lesssim & \left(\int_{t}^{t+1}\|A u(s)\|_{V^{\prime}}^{2} \mathrm{~d} s\right)^{1 / 2}+\left(\int_{t}^{t+1}\left\|B_{N}(u(s), u(s))\right\|_{V^{\prime}}^{2} \mathrm{~d} s\right)^{1 / 2}+\|g\| \\
\lesssim & \|u(s)\|_{L^{2}(t, t+1 ; V)}+\|g\|=\|S(t) u(s)\|_{L^{2}(0,1 ; V)}+\|g\| .
\end{aligned}
$$

We then obtain (3.13) from (3.19)-(3.21). Obviously the positive constant $R_{H}$ depends only on $\nu, \lambda_{1}, N$ and $\|g\|$.

Next we prove (3.14). Let $u \in \mathcal{T}_{V}^{+}$corresponding to the initial value $u_{0} \in V$. Using $A u$ to take inner product $(\cdot, \cdot)$ with equation $(2.4)$ yields the following entropy equality

$$
\frac{1}{2} \frac{\mathrm{d}}{\mathrm{d} t}\|\nabla u(t)\|^{2}+\nu\|A u(t)\|^{2}+b_{N}(u(t), u(t), A u(t))=(g, A u(t)), \forall t>0 .
$$

Recall that we have the following estimate (cf. [3, (2.7)])

$$
|b(u, v, w)| \leqslant c_{2}\|u\|_{V}^{1 / 2}\|A u\|^{1 / 2}\|v\|_{V}\|w\|, \forall u \in D(A), v \in V, w \in H .
$$

By Young's inequality,

$$
\begin{aligned}
\left|b_{N}(u, u, A u)\right| & \leqslant c_{2} \frac{N}{\|u\|_{V}}\|u\|_{V}^{3 / 2}\|A u\|^{3 / 2} \lesssim \frac{\nu}{4}\|A u\|^{2}+\|u\|_{V}^{2}, \\
|(g, A u(t))| & \lesssim \frac{\nu}{4}\|A u\|^{2}+\|g\|^{2} .
\end{aligned}
$$


Notice that $\|\nabla u\|$ is a norm in $V$ equivalent to the usual norm $\|u\|_{V}$. We then obtain from (3.22) and (3.24)-(3.25) the following entropy inequality

$$
\frac{\mathrm{d}}{\mathrm{d} t}\|u(t)\|_{V}^{2}+\nu\|A u(t)\|^{2} \lesssim\|u\|_{V}^{2}+\|g\|^{2} .
$$

Note also that the entropy inequality (3.26) is interpreted in the following sense

$$
-\int_{0}^{T}\|u(t)\|_{V}^{2} \psi^{\prime}(t) \mathrm{d} t+\nu \int_{0}^{T}\|A u(t)\|^{2} \psi(t) \mathrm{d} t \lesssim \int_{0}^{T}\left(\|g\|^{2}+\|u(t)\|_{V}^{2}\right) \psi(t) \mathrm{d} t,
$$

for any $T>0$ and any $\psi \in \mathcal{C}_{0}^{\infty}([0, T])$. Hence, using the Poincaré type inequality $\|A u(t)\|^{2} \geqslant \lambda_{1}\|u(t)\|_{V}^{2}$,

$$
\begin{aligned}
& -\int_{0}^{+\infty}\|u(t)\|_{V}^{2} \psi^{\prime}(t) \mathrm{d} t+\gamma \int_{0}^{+\infty}\|u(t)\|_{V}^{2} \psi^{\prime}(t) \mathrm{d} t \\
\lesssim & -\int_{0}^{+\infty}\left(\|A u(t)\|^{2}-\lambda_{1}\|u(t)\|_{V}^{2}\right] \psi(t) \mathrm{d} t+\int_{0}^{+\infty}\left(\|g\|^{2}+\|u(t)\|_{V}^{2}\right) \psi(t) \mathrm{d} t \\
\lesssim & \int_{0}^{+\infty}\left(\|g\|^{2}+\|u(t)\|_{V}^{2}\right) \psi(t) \mathrm{d} t, \forall \psi \in \mathcal{C}_{0}^{\infty}\left(\mathbb{R}_{+}\right) .
\end{aligned}
$$

Again by Lemma 2.4,

$$
\|u(t)\|_{V}^{2} e^{\gamma t}-\|u(\tau)\|_{V}^{2} e^{\gamma \tau} \lesssim \int_{\tau}^{t}\left(\|g\|^{2}+\|u(s)\|_{V}^{2}\right) e^{\gamma s} \mathrm{~d} s, \forall t \geqslant \tau, t, \tau \in \mathbb{R}_{+},
$$

which gives

$$
\begin{aligned}
\|u(t)\|_{V}^{2} & \lesssim\|u(\tau)\|_{V}^{2} e^{\gamma \tau} e^{-\gamma t}+e^{-\gamma t} \int_{\tau}^{t}\left(\|g\|^{2}+\|u(s)\|_{V}^{2}\right) e^{\gamma s} \mathrm{~d} s \\
& \lesssim\|u\|_{L^{\infty}(0,1 ; V)}^{2} e^{-\gamma t}+\|g\|^{2}+\int_{\tau}^{t}\|u(s)\|_{V}^{2} e^{\gamma s} \mathrm{~d} s .
\end{aligned}
$$

Inserting the following inequality (see $[3,(4.41)]$ )

$$
\|u(t)\|_{V}^{2} \leqslant\left(\left\|u_{0}\right\|_{V}^{2}+C^{(N)} t\left\|u_{0}\right\|^{2}\right) e^{-\gamma t}+\frac{\|g\|^{2}}{\nu^{2} \lambda_{1}}\left(2+C^{(N)} \nu^{2} \lambda_{1}\right)
$$

into (3.27) yields

$$
\begin{aligned}
\|u(t)\|_{V}^{2} \lesssim & \|u\|_{L^{\infty}(0,1 ; V)}^{2} e^{-\gamma t}+\|g\|^{2} \\
& +e^{-\gamma t} \int_{\tau}^{t}\left(\left(\|u\|_{L^{\infty}(0,1 ; V)}^{2}+s\|u\|_{L^{\infty}(0,1 ; V)}^{2}\right) e^{-\gamma s}+\|g\|^{2}\right) e^{\gamma s} \mathrm{~d} s \\
\lesssim & \|u\|_{L^{\infty}(0,1 ; V)}^{2}\left(1+t+t^{2}\right) e^{-\gamma t}+R_{V}^{2}, \quad \forall t \geqslant \tau \geqslant 0
\end{aligned}
$$

where $R_{V}$ is a positive constant depends only on $\nu, \lambda_{1}, c_{2}, N$ and $\|g\|$. Now by (3.16), (3.20), (3.26) and (3.28),

$$
\begin{aligned}
\|S(t) u(s)\|_{L^{2}(0,1 ; D(A))}^{2} & =\int_{t}^{t+1}\|u(s)\|_{D(A)}^{2} \mathrm{~d} s \lesssim \int_{t}^{t+1}\|A u(s)\| \mathrm{d} s \\
& \lesssim\left(\|u(t)\|_{V}^{2}+\|g\|^{2}+\int_{t}^{t+1}\|u(s)\|_{V}^{2} \mathrm{~d} s\right) \\
& \lesssim\|u(t)\|_{V}^{2}+\|g\|^{2}+\left(\|u\|_{L^{\infty}(0,1 ; H)}^{2} e^{-\gamma t}+R_{H}^{2}\right) \\
& \lesssim\|u\|_{L^{\infty}(0,1 ; V)}^{2}\left(1+t+t^{2}\right) e^{-\gamma t}+R_{V}^{2}, \quad \forall t \geqslant 1 .
\end{aligned}
$$


We then deduce from (3.12) and (3.29) that

$$
\begin{aligned}
\left\|S(t) B_{N}(u(s), u(s))\right\|_{L^{2}(0,1 ; H)}^{2} & =\int_{t}^{t+1}\left\|B_{N}(u(s), u(s))\right\|^{2} \mathrm{~d} s \lesssim \int_{t}^{t+1}\|u(s)\|_{D(A)}^{2} \mathrm{~d} s \\
& \lesssim\|u\|_{L^{\infty}(0,1 ; V)}^{2}\left(1+t+t^{2}\right) e^{-\gamma t}+R_{V}^{2}, \quad \forall t \geqslant 1 .
\end{aligned}
$$

Taking (2.4) and (3.28)-(3.30) into account, we have

$$
\begin{aligned}
\left\|S(t) \partial_{s} u(s)\right\|_{L^{2}(0,1 ; H)} & \lesssim\|S(t) A u(s)\|_{L^{2}(0,1 ; H)}+\left\|S(t) B_{N}(u(s), u(s))\right\|_{L^{2}(0,1 ; H)}+\|g\| \\
& \lesssim\|u\|_{L^{\infty}(0,1 ; V)}\left(1+t+t^{2}\right)^{1 / 2} e^{-\frac{\gamma t}{2}}+R_{V}, \quad \forall t \geqslant 1 .
\end{aligned}
$$

Therefore, (3.14) is obtained from (3.28), (3.29) and (3.31). The proof of Lemma 3.3 is completed.

We next use the estimates obtained in Lemma 3.3 to construct the trajectory attracting set for $\{S(t)\}_{t \geqslant 0}$ in $\mathcal{T}_{H}^{+}$and $\mathcal{T}_{V}^{+}$, respectively.

Lemma 3.4. Suppose $g \in H$.

(1) There exists a bounded (in the norm of $L^{\infty}\left(\mathbb{R}_{+} ; H\right)$ ) trajectory absorbing set $\Lambda_{H} \subset$ $\mathcal{T}_{H}^{+}$, i.e., for any bounded (in the norm of $L^{\infty}\left(\mathbb{R}_{+} ; H\right)$ ) subset $\mathcal{B}_{H} \subset \mathcal{T}_{H}^{+}$, there exists a time $t_{H}=t_{H}(\mathcal{B})$ such that $S(t) u \in \Lambda_{H}, \forall u \in \mathcal{B}_{H}, \forall t \geqslant t_{H}$.

(2) There exists a bounded (in the norm of $L^{\infty}\left(\mathbb{R}_{+} ; V\right)$ ) trajectory absorbing set $\Lambda_{V} \subset$ $\mathcal{T}_{V}^{+}$, i.e., for any bounded (in the norm of $L^{\infty}\left(\mathbb{R}_{+} ; V\right)$ ) subset $\mathcal{B}_{V} \subset \mathcal{T}_{V}^{+}$, there exists a time $t_{V}=t_{V}(\mathcal{B})$ such that $S(t) u \in \Lambda_{V}, \forall u \in \mathcal{B}_{V}, \forall t \geqslant t_{V}$.

Proof. Set

$$
\begin{aligned}
& \Lambda_{H}=\left\{u \in \mathcal{T}_{H}^{+} \mid \sup _{t \geqslant 0}\left\{\|u\|_{L^{\infty}(t, t+1 ; H)}+\left\|\partial_{s} u\right\|_{L^{2}\left(t, t+1 ; V^{\prime}\right)}\right\} \leqslant 2 R_{H}\right\}, \\
& \Lambda_{V}=\left\{u \in \mathcal{T}_{V}^{+} \mid \sup _{t \geqslant 0}\left\{\|u\|_{L^{\infty}(t, t+1 ; V)}+\left\|\partial_{s} u\right\|_{L^{2}(t, t+1 ; H)}\right\} \leqslant 2 R_{V}\right\},
\end{aligned}
$$

where $R_{H}$ and $R_{V}$ are the positive constants come from Lemma 3.3.

We claim that $\Lambda_{H}$ is a bounded trajectory absorbing set (thus is a bounded trajectory attracting set) for $\{S(t)\}_{t \geqslant 0}$ in $\mathcal{T}_{H}^{+}$. Indeed, let $\mathcal{B}_{H}$ be a bounded (in $L^{\infty}\left(\mathbb{R}_{+} ; H\right)$ norm) subset of $\mathcal{T}_{H}^{+}$. Then from (3.13) we infer that for $\forall u \in \mathcal{B}_{H} \subset \mathcal{T}_{H}^{+}$, there exists a $t_{0}=t_{0}\left(\mathcal{B}_{H}\right) \geqslant 1$ such that $\|u\|_{L^{\infty}(0,1 ; H)} e^{-\gamma t / 2} \leqslant R_{H}$ as long as $t \geqslant t_{0}$. Therefore,

$$
\|u\|_{L^{\infty}(t, t+1 ; H)}+\left\|\partial_{t} u\right\|_{L^{2}\left(t, t+1 ; V^{\prime}\right)} \leqslant 2 R_{H}, \quad \forall t \geqslant t_{0}+1
$$

and $S(t) \mathcal{B}_{H} \subseteq \Lambda_{H}, \forall t \geqslant t_{H}=t_{0}+1$, which implies that $\Lambda_{H}$ is a trajectory absorbing set for $\{S(t)\}_{t \geqslant 0}$ in $\mathcal{T}_{H}^{+}$. Obviously, $\Lambda_{H}$ is bounded (in $L^{\infty}\left(\mathbb{R}_{+} ; H\right)$ norm) in $\mathcal{T}_{H}^{+}$.

Similarly, $\Lambda_{V}$ is a bounded trajectory absorbing set (thus is a bounded trajectory attracting set) for $\{S(t)\}_{t \geqslant 0}$ in $\mathcal{T}_{V}^{+}$. The proof is completed.

To prove the existence of trajectory attractor for $\{S(t)\}_{t \geqslant 0}$ in the trajectory space $\mathcal{T}_{H}^{+}$, we now need establish that the trajectory absorbing set $\Lambda_{H}$ is compact in $\mathcal{C}_{\text {loc }}\left(\mathbb{R}_{+} ; H^{-\eta}\right)$. 
Lemma 3.5. Suppose $g \in H$. Then the trajectory absorbing set $\Lambda_{H}$ is compact in $\mathcal{C}_{\text {loc }}\left(\mathbb{R}_{+} ; H^{-\eta}\right)$.

Proof. Step One We prove that $\mathcal{T}_{H}^{+}$is closed in $\mathcal{C}_{\text {loc }}\left(\mathbb{R}_{+} ; H^{-\eta}\right)$. Let $\left\{u_{n}\right\}$ be a bounded (in the norm of $L^{\infty}\left(\mathbb{R}_{+} ; H\right)$ ) sequence in $\mathcal{T}_{H}^{+}$and there exists a function $u^{*} \in \mathcal{C}_{\text {loc }}\left(\mathbb{R}_{+} ; H^{-\eta}\right)$ such that

$$
u_{n} \longrightarrow u^{*} \text { in the topology of } \mathcal{C}_{\text {loc }}\left(\mathbb{R}_{+} ; H^{-\eta}\right) \text { as } n \rightarrow \infty \text {. }
$$

We shall prove that $u^{*} \in \mathcal{T}_{H}^{+}$. To this end, we need to show that $u^{*} \in L^{\infty}\left(\mathbb{R}_{+} ; H\right) \cap$ $L_{l o c}^{2}\left(\mathbb{R}_{+} ; V\right)$, and $\forall T>0, \Pi_{T} u^{*}(t)$ is a weak solution of $(2.4)$ on the interval $[0, T]$. Indeed, since $\left\{u_{n}\right\} \subset \mathcal{T}_{H}^{+}$and is bounded in $L^{\infty}\left(\mathbb{R}_{+} ; H\right)$, by (3.13) we conclude that $\left\{u_{n}\right\}$ is bounded in $L_{\text {loc }}^{2}\left(\mathbb{R}_{+} ; V\right)$ and $\left\{\partial_{t} u_{n}\right\}$ is bounded in $L_{l o c}^{2}\left(\mathbb{R}_{+} ; V^{\prime}\right)$. Using the diagonal procedure we deduce that there exist a function $u \in L^{\infty}\left(\mathbb{R}_{+} ; H\right) \cap L_{\text {loc }}^{2}\left(\mathbb{R}_{+} ; V\right)$ and a subsequence $\left\{u_{n^{\prime}}\right\}$ of $\left\{u_{n}\right\}$ such that

$$
\begin{aligned}
& \Pi_{T} u_{n^{\prime}} \rightarrow \Pi_{T} u \text { weakly in } L^{2}(0, T ; V) \text { as } n^{\prime} \rightarrow \infty, \\
& u_{n^{\prime}} \rightarrow u \text { weakly star in } L^{\infty}\left(\mathbb{R}_{+} ; H\right) \text { as } n^{\prime} \rightarrow \infty, \\
& \partial_{t} \Pi_{T} u_{n^{\prime}} \rightarrow \partial_{t} \Pi_{T} u \text { weakly in } L^{2}\left(0, T ; V^{\prime}\right) \text { as } n^{\prime} \rightarrow \infty .
\end{aligned}
$$

Obviously, $\partial_{t} u \in L_{l o c}^{2}\left(\mathbb{R}_{+} ; V^{\prime}\right)$. By Lemma 2.3 we obtain $\Pi_{T} u \in \mathcal{C}\left([0, T] ; H^{-\eta}\right)$ since the embedding $H \hookrightarrow H^{-\eta}$ is compact. From (3.35) and the uniqueness of limit we have $u=u^{*}$. Next we verify that $\Pi_{T} u^{*}$ is a weak solution of $(2.4)$ on the interval $[0, T]$. For this purpose, we check the following convergent relations

$$
\begin{aligned}
& A \Pi_{T} u_{n^{\prime}} \rightarrow A \Pi_{T} u^{*} \text { weakly in } L^{2}\left(0, T ; V^{\prime}\right) \text { as } n^{\prime} \rightarrow \infty, \\
& B_{N}\left(\Pi_{T} u_{n^{\prime}}, \Pi_{T} u_{n^{\prime}}\right) \rightarrow B_{N}\left(\Pi_{T} u^{*}, \Pi_{T} u^{*}\right) \text { weakly in } L^{2}\left(0, T ; V^{\prime}\right) \text { as } n^{\prime} \rightarrow \infty .
\end{aligned}
$$

In fact, the convergence of (3.39) is classical (see e.g. [28]), while the derivation of (3.40) is almost the same as that as $[3,(3.20)]$. With the convergent relations (3.38)-(3.40) in hand, we can pass the limit in equation (2.4) as $n^{\prime} \rightarrow \infty$. This shows that $\Pi_{T} u^{*}$ is a weak solution of equation $(2.4)$ on $[0, T]$.

Step Two We prove that the trajectory absorbing set $\Lambda_{H}$ is compact in $\mathcal{C}_{\text {loc }}\left(\mathbb{R}_{+} ; H^{-\eta}\right)$. Indeed, from (3.32) we see that $\Pi_{T} \Lambda_{H}$ is bounded in $W_{\infty, 2}\left(0, T ; H, V^{\prime}\right)$ and thus $\Pi_{T} \Lambda_{H}$ is pre-compact in $\mathcal{C}\left([0, T] ; H^{-\eta}\right)$ (thanks to Lemma 2.3). Hence, it suffices to show that $\Pi_{T} \Lambda_{H}$ is closed in $\mathcal{C}\left([0, T] ; H^{-\eta}\right)$ for any $T>0$. Assume that $\left\{u_{n}\right\} \subset \Lambda_{H}$ and $\Pi_{T} u_{n} \longrightarrow \Pi_{T} u$ in the metric of $\mathcal{C}\left([0, T] ; H^{-\eta}\right)$ as $n \rightarrow \infty$. we see from Step one that $u \in \mathcal{T}_{H}^{+}$. Moreover, in the proof of Step one we know $\partial_{t} \Pi_{T} u_{n} \rightarrow \partial_{t} \Pi_{T} u$ weakly in $L^{2}\left(0, T ; V^{\prime}\right)$ and $u_{n} \rightarrow u$ weakly star in $L^{\infty}\left(\mathbb{R}_{+} ; H\right)$ as $n \rightarrow \infty$. Thus

$$
\begin{aligned}
& \|u\|_{L^{\infty}(t, t+1 ; H)}+\left\|\partial_{t} u\right\|_{L^{2}\left(t, t+1 ; V^{\prime}\right)} \\
\leqslant & \liminf _{n \rightarrow \infty}\left\|u_{n}\right\|_{L^{\infty}(t, t+1 ; H)}+\liminf _{n \rightarrow \infty}\left\|\partial_{t} u_{n}\right\|_{L^{2}\left(t, t+1 ; V^{\prime}\right)} \leqslant 2 R_{H}, \forall t \geqslant 0 .
\end{aligned}
$$

Therefore $u \in \Lambda_{H}$ and $\Pi_{T} u \in \mathcal{C}\left([0, T] ; H^{-\eta}\right)$. The proof is completed.

Similarly, in order to obtain the existence of trajectory attractor for $\{S(t)\}_{t \geqslant 0}$ in the regular trajectory space $\mathcal{T}_{V}^{+}$, we need prove that the trajectory absorbing set $\Lambda_{V}$ is compact in $\mathcal{C}_{\text {loc }}\left(\mathbb{R}_{+} ; H^{1-\eta}\right)$. 
Lemma 3.6. Suppose $g \in H$. Then the trajectory absorbing set $\Lambda_{V}$ is compact in $\mathcal{C}_{\text {loc }}\left(\mathbb{R}_{+} ; H^{1-\eta}\right)$.

Proof. The procedure of the proof is analogous to that of Lemma 3.5, which is divided into two steps.

Step One We prove that $\mathcal{T}_{V}^{+}$is closed in $\mathcal{C}_{\text {loc }}\left(\mathbb{R}_{+} ; H^{1-\eta}\right)$. Let $\left\{u_{n}\right\}$ be a bounded (in the norm of $\left.L^{\infty}\left(\mathbb{R}_{+} ; V\right)\right)$ sequence in $\mathcal{T}_{V}^{+}$and there exists a function $u^{*} \in \mathcal{C}_{\text {loc }}\left(\mathbb{R}_{+} ; H^{1-\eta}\right)$ such that

$$
u_{n} \longrightarrow u^{*} \text { in the topology of } \mathcal{C}_{l o c}\left(\mathbb{R}_{+} ; H^{1-\eta}\right) \text { as } n \rightarrow \infty \text {. }
$$

We next prove that $u^{*} \in \mathcal{T}_{V}^{+}$. To this end, we need to show that $u^{*} \in L^{\infty}\left(\mathbb{R}_{+} ; V\right) \cap$ $L_{\text {loc }}^{2}\left(\mathbb{R}_{+} ; D(A)\right)$, and $\forall T>0, \Pi_{T} u^{*}(t)$ is a strong solution of $(2.4)$ on the interval $[0, T]$. In fact, since $\left\{u_{n}\right\} \subset \mathcal{T}_{V}^{+}$and is bounded in $L^{\infty}\left(\mathbb{R}_{+} ; V\right)$, by (3.14) we conclude that $\left\{u_{n}\right\}$ is bounded in $L_{l o c}^{2}\left(\mathbb{R}_{+} ; D(A)\right)$ and $\left\{\partial_{t} u_{n}\right\}$ is bounded in $L_{l o c}^{2}\left(\mathbb{R}_{+} ; H\right)$. Using the diagonal procedure we see that there exist a function $u \in L^{\infty}\left(\mathbb{R}_{+} ; V\right) \cap L_{l o c}^{2}\left(\mathbb{R}_{+} ; D(A)\right)$ and a subsequence $\left\{u_{n^{\prime}}\right\}$ of $\left\{u_{n}\right\}$ such that

$$
\begin{aligned}
& \Pi_{T} u_{n^{\prime}} \rightarrow \Pi_{T} u \text { weakly in } L^{2}(0, T ; D(A)) \text { as } n^{\prime} \rightarrow \infty, \\
& u_{n^{\prime}} \rightarrow u \text { weakly star in } L^{\infty}\left(\mathbb{R}_{+} ; V\right) \text { as } n^{\prime} \rightarrow \infty, \\
& \partial_{t} \Pi_{T} u_{n^{\prime}} \rightarrow \partial_{t} \Pi_{T} u \text { weakly in } L^{2}(0, T ; H) \text { as } n^{\prime} \rightarrow \infty .
\end{aligned}
$$

Obviously, $\partial_{t} u \in L_{\text {loc }}^{2}\left(\mathbb{R}_{+} ; H\right)$. By Lemma 2.3 we have $\Pi_{T} u \in \mathcal{C}\left([0, T] ; H^{1-\eta}\right)$ since the embedding $V \hookrightarrow H^{1-\eta}$ is compact. From (3.41) and the uniqueness of limit we have $u=u^{*}$. Next we verify that $\Pi_{T} u^{*}$ is a strong solution of $(2.4)$ on the interval $[0, T]$. Since we have the following convergent relations

$$
\begin{aligned}
& A \Pi_{T} u_{n^{\prime}} \rightarrow A \Pi_{T} u^{*} \text { weakly in } L^{2}(0, T ; H) \text { as } n^{\prime} \rightarrow \infty \\
& B_{N}\left(\Pi_{T} u_{n^{\prime}}, \Pi_{T} u_{n^{\prime}}\right) \rightarrow B_{N}\left(\Pi_{T} u^{*}, \Pi_{T} u^{*}\right) \text { weakly in } L^{2}(0, T ; H) \text { as } n^{\prime} \rightarrow \infty
\end{aligned}
$$

which were proved in [3], we can pass the limit in equation (2.4) as $n^{\prime} \rightarrow \infty$ and conclude that $\Pi_{T} u^{*}$ is a strong solution of equation $(2.4)$ on $[0, T]$.

Step Two We prove that the trajectory absorbing set $\Lambda_{V}$ is compact in $\mathcal{C}_{\text {loc }}\left(\mathbb{R}_{+} ; H^{1-\eta}\right)$. From (3.33) we see that $\Pi_{T} \Lambda_{H}$ is bounded in $W_{\infty, 2}(0, T ; V, H)$. Thus $\Pi_{T} \Lambda_{V}$ is precompact in $\mathcal{C}\left([0, T] ; H^{1-\eta}\right)$ due to Lemma 2.3. Hence, it suffices to show that $\Pi_{T} \Lambda_{V}$ is closed in $\mathcal{C}\left([0, T] ; H^{1-\eta}\right)$ for any $T>0$. Assume that $\left\{u_{n}\right\} \subset \Lambda_{V}$ and $\Pi_{T} u_{n} \longrightarrow \Pi_{T} u$ in the metric of $\mathcal{C}\left([0, T] ; H^{1-\eta}\right)$ as $n \rightarrow \infty$. Then $u \in \mathcal{T}_{V}^{+}$. Moreover, $\partial_{t} \Pi_{T} u_{n} \rightarrow \partial_{t} \Pi_{T} u$ weakly in $L^{2}(0, T ; H)$ and $u_{n} \rightarrow u$ weakly star in $L^{\infty}\left(\mathbb{R}_{+} ; V\right)$ as $n \rightarrow \infty$. Thus

$$
\begin{aligned}
& \|u\|_{L^{\infty}(t, t+1 ; V)}+\left\|\partial_{t} u\right\|_{L^{2}(t, t+1 ; H)} \\
\leqslant & \liminf _{n \rightarrow \infty}\left\|u_{n}\right\|_{L^{\infty}(t, t+1 ; V)}+\liminf _{n \rightarrow \infty}\left\|\partial_{t} u_{n}\right\|_{L^{2}(t, t+1 ; H)} \leqslant 2 R_{V}, \forall t \geqslant 0 .
\end{aligned}
$$

Therefore $u \in \Lambda_{V}$ and $\Pi_{T} u \in \mathcal{C}\left([0, T] ; H^{1-\eta}\right)$. The proof is completed.

Remember that we have introduced the notation $\mathcal{K}_{X}$ with $X=H$ or $X=V$ in the Introduction. The main result of this section reads as follows. 
Theorem 3.1. Suppose $g \in H$.

(1) The natural translation semigroup $\{S(t)\}_{t \geqslant 0}$ possesses a trajectory attractor $\mathcal{A}_{H}^{\mathrm{tr}}$ in $\mathcal{T}_{H}^{+}$satisfying Definition 3.2(1)(i)-(iii) and possessing the following structure

$$
\mathcal{A}_{H}^{\mathrm{tr}}=\Pi_{+} \mathcal{K}_{H}=\left\{\Pi_{+} u(\cdot) \mid u \in \mathcal{K}_{H}\right\} \subset \mathcal{T}_{H}^{+} .
$$

(2) The natural translation semigroup $\{S(t)\}_{t \geqslant 0}$ possesses a trajectory attractor $\mathcal{A}_{V}^{\mathrm{tr}}$ in $\mathcal{T}_{V}^{+}$satisfying Definition 3.2(2)(i)-(iii) and possessing the following structure

$$
\mathcal{A}_{V}^{\mathrm{tr}}=\Pi_{+} \mathcal{K}_{V}=\left\{\Pi_{+} u(\cdot) \mid u \in \mathcal{K}_{V}\right\} \subset \mathcal{T}_{V}^{+} .
$$

(3) The following regularity of the trajectory attractor holds

$$
\mathcal{A}_{H}^{\mathrm{tr}}=\Pi_{+} \mathcal{K}_{H}=\mathcal{A}_{V}^{\mathrm{tr}}=\Pi_{+} \mathcal{K}_{V} .
$$

Proof. The results in items (1) and (2) are obtained directly by employing Lemma 3.1, Lemmas 3.4-3.6 and the abstract theory [31, Theorem 4.1].

We next prove (3.49). To this end, we first establish that any trajectory $u(\cdot)$ issuing from any bounded subset of $H$ will becomes eventually bounded in $L^{\infty}\left(\mathbb{R}_{+} ; V\right)$. Indeed, consider any bounded subset $\mathcal{B}_{H}$ of $H$ and any $u_{0} \in \mathcal{B}_{H}$. Let $u(\cdot)=S(\cdot) u_{0}$ be a trajectory issuing from $u_{0}$. Then by Lemma $2.2(1)$, there exists some $t_{0} \in(0,1)$ such that $u\left(t_{0}\right) \in V$. Then the function $v(t)=u\left(t+t_{0}\right)$ is a weak solution of equation (2.4) with initial value $v(0)=u\left(t_{0}\right) \in V$. By [3, (4.41)] we have

$$
\begin{aligned}
\left\|u\left(t+t_{0}\right)\right\|_{V}^{2}=\|v(t)\|_{V}^{2} & \lesssim\left(\|v(0)\|_{V}^{2}+t\|v(0)\|^{2}\right) e^{-\sigma t}+\|g\| \\
& =\left(\left\|u\left(t_{0}\right)\right\|_{V}^{2}+t\left\|u\left(t_{0}\right)\right\|^{2}\right) e^{-\sigma t}+\|g\| .
\end{aligned}
$$

Set

$$
\Psi=\left\{u(\cdot) \in \mathcal{T}_{H}^{+} \mid u(\cdot) \text { issues from } u_{0} \in \mathcal{B}_{H}\right\} .
$$

Then for above $t_{0}$ we see that both $\sup _{u(\cdot) \in \Psi}\left\|u\left(t_{0}\right)\right\|_{V}$ and $\sup _{u(\cdot) \in \Psi}\left\|u\left(t_{0}\right)\right\|$ depend only on $\mathcal{B}_{H}$. We then deduce from (3.50) that there exists some time $t^{*}\left(\mathcal{B}_{H}\right)>t_{0}$ such that

$$
\|u(t)\|_{V}^{2} \leqslant c, \forall t>t^{*}\left(\mathcal{B}_{H}\right),
$$

where the positive constant $c$ depends only on $\|g\|$ and $\mathcal{B}_{H}$. Now, (3.51) and the invariance property of the trajectory attractor tell us that there is some time $t^{*}\left(\mathcal{A}_{H}^{\mathrm{tr}}\right)$ such that $\mathcal{A}_{H}^{\mathrm{tr}}=S\left(t^{*}\left(\mathcal{A}_{H}^{\mathrm{tr}}\right)\right) \mathcal{A}_{H}^{\mathrm{tr}}$ is bounded in $L^{\infty}\left(\mathbb{R}_{+} ; V\right)$. Also by the invariance property and attracting property of the trajectory attractor, we obtain for any $T>0$ that

$$
\begin{aligned}
& \operatorname{dist}_{\mathcal{C}\left([0, T] ; H^{-\eta}\right)}\left(\Pi_{T} \mathcal{A}_{H}^{\mathrm{tr}}, \Pi_{T} \mathcal{A}_{V}^{\mathrm{tr}}\right) \\
= & \operatorname{dist}_{\mathcal{C}\left([0, T] ; H^{-\eta}\right)}\left(\Pi_{T} S(t) \mathcal{A}_{H}^{\mathrm{tr}}, \Pi_{T} \mathcal{A}_{V}^{\mathrm{tr}}\right) \quad\left(\forall t \in \mathbb{R}_{+}\right) \\
\leqslant & \operatorname{dist}_{\mathcal{C}\left([0, T] ; H^{1-\eta}\right)}\left(\Pi_{T} S(t) \mathcal{A}_{H}^{\mathrm{tr}}, \Pi_{T} \mathcal{A}_{V}^{\mathrm{tr}}\right) \quad\left(\forall t \geqslant t^{*}\left(\mathcal{A}_{H}^{\mathrm{tr}}\right)\right) \\
= & \lim _{t \rightarrow+\infty} \operatorname{dist}_{\mathcal{C}\left([0, T] ; H^{1-\eta}\right)}\left(\Pi_{T} S(t) \mathcal{A}_{H}^{\mathrm{tr}}, \Pi_{T} \mathcal{A}_{V}^{\mathrm{tr}}\right) \\
= & 0,
\end{aligned}
$$


which implies $\mathcal{A}_{H}^{\mathrm{tr}} \subseteq \mathcal{A}_{V}^{\mathrm{tr}}$. At the same time, since all strong solutions are weak solutions, (3.47) and (3.48) give $\mathcal{A}_{V}^{\text {tr }} \subseteq \mathcal{A}_{H}^{\text {tr }}$. The proof is completed.

\section{Invariant measure and trajectory statistical solution for the 3D globally modified Navier-Stokes equations}

In this section we will investigate the existence and uniqueness of invariant Borel probability measure and trajectory statistical solution for the 3D globally modified Navier-Stokes equations. Our idea originates from [6]. Here we extend the approach of [6] and use the trajectory attractor to construct the invariant measure and trajectory statistical solution.

According to [2, Definition 3.1], we now give the concept of the trajectory statistical solution for the addressed 3D globally modified Navier-Stokes equations. For brevity, we denote

$$
\mathcal{F}^{+}=\mathcal{C}\left([0,+\infty) ; H^{-\eta}\right),
$$

which has been endowed with the metric (see (3.1))

$$
\mathrm{d}_{\mathcal{F}^{+}}(u, v)=\mathrm{d}_{\mathcal{C}\left([0,+\infty) ; H^{-\eta}\right)}(u, v)=\sum_{T \in \mathbb{N}} \frac{1}{2^{T}} \frac{\sup \left\{\mathrm{d}_{H^{-\eta}}(u(t), v(t)): 0 \leqslant t \leqslant T\right\}}{1+\sup \left\{\mathrm{d}_{H^{-\eta}}(w(t), \phi(t)): 0 \leqslant t \leqslant T\right\}} .
$$

Definition 4.1. We say a Borel probability measure $\rho$ on $\mathcal{F}^{+}$is a $\mathcal{T}_{H}^{+}$-trajectory statistical solution over $[0,+\infty)$ (or simply a trajectory statistical solution) for equations (1.1)-(1.2) if

(1) $\rho$ is tight for any $\mathcal{B} \in \mathcal{B}\left(\mathcal{F}^{+}\right)$(the collection of Borel sets of $\mathcal{F}^{+}$) in the sense that

$$
\rho(\mathcal{B})=\sup \left\{\rho(E) \mid E \in \mathcal{B}\left(\mathcal{F}^{+}\right) \text {and } E \subset \mathcal{B}\right\} ;
$$

(2) $\rho$ is supported by a Borel subset of $\mathcal{F}^{+}$included in $\mathcal{T}_{H}^{+}$.

To construct the invariant Borel probability measure and trajectory statistical solution, we first establish two lemmas. Let $\mathcal{C}\left(\mathcal{F}^{+}\right)$be the set of all continuous functions from $\mathcal{F}^{+}$to $\mathbb{R}$. For any $\epsilon>0$, denote $K_{\epsilon}=\left\{w \in \mathcal{F}^{+} \mid \inf _{v \in K} \mathrm{~d}_{\mathcal{F}^{+}}(w, v)<\epsilon\right\}$.

Lemma 4.1. Let $K$ be some compact subset of $\mathcal{F}^{+}$. Then for every $\psi \in \mathcal{C}\left(\mathcal{F}^{+}\right)$, there exists some $\epsilon>0$ such that

$$
\sup _{w \in K_{\epsilon}}|\psi(w)|<+\infty \text {. }
$$

Proof. Fix some $\psi \in \mathcal{C}\left(\mathcal{F}^{+}\right)$. For every $w \in K$ we can choose $\delta=\delta_{w}>0$ such that for every $v \in \mathcal{B}\left(w ; \delta_{w}\right)=\left\{\Phi \in \mathcal{F}^{+} \mid \mathrm{d}_{\mathcal{F}^{+}}(w, \Phi)<\delta_{w}\right\}$ there holds $\mid \psi(w)-$ $\psi(v) \mid<1$. Picking numbers $\delta_{w}>0$ in this way we can construct an open covering $\Lambda=\left\{\mathcal{B}\left(w ; \frac{\delta_{w}}{3}\right) \mid w \in K\right\}$ for $K$. Since $K$ is compact in $\mathcal{F}^{+}$, we may extract from this open covering a finite one

$$
\Lambda_{m}=\left\{\mathcal{B}\left(w_{1} ; \frac{\delta_{w_{1}}}{3}\right), \mathcal{B}\left(w_{2} ; \frac{\delta_{w_{2}}}{3}\right), \cdots, \mathcal{B}\left(w_{m} ; \frac{\delta_{w_{m}}}{3}\right)\right\}
$$


Set

$$
\epsilon=\frac{\min \left\{\delta_{w_{1}}, \delta_{w_{2}}, \cdots, \delta_{w_{m}}\right\}}{3}, \quad C=1+\max _{1 \leqslant j \leqslant m}\left|\psi\left(w_{j}\right)\right| .
$$

Given any $w \in K_{\epsilon}$ we can choose $v \in K$ so that $\mathrm{d}_{\mathcal{F}^{+}}(w, v)<2 \epsilon$. Since $\Lambda_{m}$ covers $K$ we can choose $w_{j}$ such that $\mathrm{d}_{\mathcal{F}^{+}}\left(v, w_{j}\right)<\frac{\delta_{w_{j}}}{3}$. Hence we obtain

$$
\mathrm{d}_{\mathcal{F}^{+}}\left(w, w_{j}\right)<\mathrm{d}_{\mathcal{F}^{+}}(w, v)+\mathrm{d}_{\mathcal{F}^{+}}\left(v, w_{j}\right)<2 \epsilon+\frac{\delta_{w_{j}}}{3} \leqslant \delta_{w_{j}}
$$

and conclude that $|\psi(w)| \leqslant C$. By the arbitrariness of $w \in K_{\epsilon}$, we end the proof.

Lemma 4.2. Let $K$ be some compact subset of $\mathcal{F}^{+}$and let $\psi, \phi \in \mathcal{C}\left(\mathcal{F}^{+}\right)$satisfying $\psi(w)=\phi(w)$ for every $w \in K$. Then for every $\epsilon>0$ there exists a $\delta=\delta(\epsilon)>0$ such that $\sup _{w \in K_{\delta}}|\psi(w)-\phi(w)|<\epsilon$.

Proof. Consider given $\epsilon>0$. For every $w \in K$ we pick $\gamma_{w}>0$ so that $|\phi(w)-\phi(v)|+$ $|\psi(w)-\psi(v)|<\epsilon$ whenever $v \in \mathcal{B}\left(w ; \gamma_{w}\right)$. Due to the compactness of $K$ in $\mathcal{F}^{+}$, we can cover $K$ with a finite collection

$$
\Lambda_{k}=\left\{\mathcal{B}\left(w_{1} ; \frac{\gamma_{w_{1}}}{3}\right), \mathcal{B}\left(w_{2} ; \frac{\gamma_{w_{2}}}{3}\right), \cdots, \mathcal{B}\left(w_{k} ; \frac{\gamma_{w_{k}}}{3}\right)\right\} \text { with } w_{j} \in K, j=1,2, \cdots, k .
$$

Set $3 \delta=\min \left\{\gamma_{w_{1}}, \gamma_{w_{2}}, \cdots, \gamma_{w_{k}}\right\}$, then for every $v \in K_{\delta}$ we can choose $w \in K$ so that $\mathrm{d}_{\mathcal{F}^{+}}(v, w)<2 \delta$. Notice that $K$ is covered by $\Lambda_{k}$, we may take $w_{j}$ such that $\mathrm{d}_{\mathcal{F}^{+}}\left(w, w_{j}\right)<\frac{\gamma_{w_{j}}}{3}$. Thus

$$
\mathrm{d}_{\mathcal{F}^{+}}\left(v, w_{j}\right) \leqslant \mathrm{d}_{\mathcal{F}^{+}}(v, w)+\mathrm{d}_{\mathcal{F}^{+}}\left(w, w_{j}\right) \leqslant 2 \delta+\frac{\gamma_{w_{j}}}{3} \leqslant \gamma_{w_{j}} .
$$

Therefore for arbitrary $v \in K_{\delta}$, there exists some $j$ such that $v \in \mathcal{B}\left(w_{j} ; \gamma_{w_{j}}\right)$. Keeping in mind that $\psi\left(w_{j}\right)=\phi\left(w_{j}\right)$, we have

$$
|\psi(v)-\phi(v)| \leqslant\left|\psi(v)-\psi\left(w_{j}\right)\right|+\left|\phi\left(w_{j}\right)-\phi(v)\right|<\epsilon .
$$

The proof is completed.

We next recall the definition of generalized Banach limit and a useful property.

Definition 4.2. ( $[12,22])$ A generalized Banach limit is any linear functional, which we denote by $\mathrm{LIM}_{t \rightarrow+\infty}$, defined on the space of all bounded real-valued functions on $[0,+\infty)$ that satisfies

(1) $\operatorname{LIM}_{t \rightarrow+\infty} h(t) \geqslant 0$ for nonnegative functions $h(\cdot)$ on $[0,+\infty)$;

(2) $\operatorname{LIM}_{t \rightarrow+\infty} h(t)=\lim _{t \rightarrow+\infty} h(t)$ if the usual limit $\lim _{t \rightarrow+\infty} h(t)$ exists.

Let $B_{+}$be the collection of all bounded real-valued functions on $[0,+\infty)$. For any generalized Banach limit $\operatorname{LIM}_{t \rightarrow+\infty}$, the following useful property

$$
\left|\mathrm{LIM}_{t \rightarrow+\infty} h(t)\right| \leqslant \limsup _{t \rightarrow+\infty}|h(t)|, \quad \forall h(\cdot) \in B_{+},
$$

is presented in $[12,(1.38)]$ and in $[6,(2.3)]$.

The main result of this section reads as follows. 
Theorem 4.1. Let $\mathrm{LIM}_{t \rightarrow+\infty}$ be a given generalized Banach limit. Then for any $u \in$ $\mathcal{T}_{H}^{+}$, there exists a unique Borel probability measure $\rho_{u}$ on $\mathcal{F}^{+}$such that

$$
\int_{\mathcal{F}^{+}} \psi(v) \mathrm{d} \rho_{u}(v)=\operatorname{LIM}_{t \rightarrow+\infty} \frac{1}{t} \int_{0}^{t} \psi(S(s) u) \mathrm{d} s, \quad \forall \psi \in \mathcal{C}\left(\mathcal{F}^{+}\right) .
$$

Moreover, this Borel probability measure $\rho_{u}$ is a trajectory statistical solution for the $3 D$ globally modified Navier-Stokes equations (1.1)-(1.2) and $\rho_{u}$ satisfies the following invariant property and regularity

$$
\begin{aligned}
\int_{\mathcal{F}^{+}} \psi(S(\tau) v) \mathrm{d} \rho_{u}(v) & =\int_{\mathcal{F}^{+}} \psi(v) \mathrm{d} \rho_{u}(v)=\int_{\mathcal{A}_{H}^{\text {tr }}} \psi(v) \mathrm{d} \rho_{u}(v) \\
& =\int_{\mathcal{A}_{V}^{\text {tr }}} \psi(v) \mathrm{d} \rho_{u}(v), \quad \forall \tau \geqslant 0, \quad \forall \psi \in \mathcal{C}\left(\mathcal{F}^{+}\right) .
\end{aligned}
$$

Proof. Let $\operatorname{LIM}_{t \rightarrow+\infty}$ be a given generalized Banach limit. Consider given $u \in \mathcal{T}_{H}^{+}$and $\psi \in \mathcal{C}\left(\mathcal{F}^{+}\right)$. Since the translation semigroup $\{S(s)\}_{s \geqslant 0}$ possesses a trajectory attractor $\mathcal{A}_{H}^{\text {tr }} \subset \mathcal{T}_{H}^{+} \subset \mathcal{F}^{+}$, we see from the attracting property of the trajectory attractor that for every $\epsilon>0$, there exists a time $t_{\epsilon} \geqslant 0$ such that

$$
S(s) u \in \mathcal{A}_{H, \epsilon}^{\mathrm{tr}}=\left\{\Phi \in \mathcal{F}^{+} \mid \inf _{v \in \mathcal{A}_{H}^{\text {tr }}} \mathrm{d}_{\mathcal{F}^{+}}(\Phi, v)<\epsilon\right\}, \text { for every } s \geqslant t_{\epsilon} .
$$

By Lemma 4.1 we can pick $\epsilon>0$ such that

$$
C_{1}=\sup _{v \in \mathcal{A}_{H, \epsilon}^{\text {tr }}}|\psi(v)|<+\infty
$$

Notice that the interval $\left[0, t_{\epsilon}\right]$ is compact and that $s \longmapsto|\psi(S(s) u)|$ is continuous, we can take $t_{\epsilon}$ as required in (4.4) for the picked $\epsilon$, and see that

$$
C_{2}=\sup _{s \in\left[0, t_{\epsilon}\right]}|\psi(S(s) u)|<+\infty .
$$

Hence, we conclude for every $t>0$ that

$$
\frac{1}{t} \int_{0}^{t} \psi(S(s) u) \mathrm{d} s \leqslant C_{1}+C_{2}<+\infty,
$$

which implies that the map defined by $t \longmapsto \frac{1}{t} \int_{0}^{t} \psi(S(s) u) \mathrm{d} s$ is bounded over $[0,+\infty)$. Therefore, we claim that

$$
\mathcal{L}_{u}(\psi)=\operatorname{LIM}_{t \rightarrow+\infty} \frac{1}{t} \int_{0}^{t} \psi(S(s) u) \mathrm{d} s
$$

is well defined as a positive linear functional on $\mathcal{C}\left(\mathcal{F}^{+}\right)$. We next establish that the positive linear functional $\mathcal{L}_{u}(\psi)$ depends only on the values of $\psi$ on the trajectory attractor $\mathcal{A}_{H}^{\mathrm{tr}}$. In other words, we will prove that if $\psi(v)=\widetilde{\psi}(v)$ for every $v \in \mathcal{A}_{H}^{\mathrm{tr}}$ 
then $\mathcal{L}_{u}(\psi)=\mathcal{L}_{u}(\widetilde{\psi})$. In fact, for any given $\epsilon>0$, we can choose, by Lemma 4.2 a corresponding $\delta>0$ such that

$$
\sup _{v \in \mathcal{A}_{H, \delta}^{\mathrm{tr}}}|\psi(v)-\widetilde{\psi}(v)|<\epsilon
$$

where $\mathcal{A}_{H, \delta}^{\text {tr }}=\left\{\Phi \in \mathcal{F}^{+} \mid \inf _{v \in \mathcal{A}_{H}^{\text {tr }}} \mathrm{d}_{\mathcal{F}^{+}}(\Phi, v)<\delta\right\}$. We now pick $t_{\delta}>0$ such that $S(s) u \in$ $\mathcal{A}_{H, \delta}^{\mathrm{tr}}$ for every $s \geqslant t_{\delta}$. Set

$$
C_{\delta}=\sup _{s \in\left[0, t_{\delta}\right]}(|\psi(S(s) u)|+|\widetilde{\psi}(S(s) u)|) .
$$

Similar to (4.6), we find that $C_{\delta}<+\infty$. Applying (4.1), (4.6) and (4.8), we have

$$
\begin{aligned}
\left|\mathcal{L}_{u}(\psi-\widetilde{\psi})\right|= & \left|\operatorname{LIM}_{t \rightarrow+\infty} \frac{1}{t} \int_{0}^{t}(\psi(S(s) u)-\widetilde{\psi}(S(s) u)) \mathrm{d} s\right| \\
\leqslant & \limsup _{t \rightarrow+\infty} \frac{1}{t}\left|\int_{0}^{t}(\psi(S(s) u)-\widetilde{\psi}(S(s) u)) \mathrm{d} s\right| \\
\leqslant & \limsup _{t \rightarrow+\infty} \frac{1}{t} \int_{0}^{t_{\delta}}|\psi(S(s) u)-\widetilde{\psi}(S(s) u)| \mathrm{d} s \\
& +\limsup _{t \rightarrow+\infty} \frac{1}{t} \int_{t_{\delta}}^{t}|\psi(S(s) u)-\widetilde{\psi}(S(s) u)| \mathrm{d} s \\
\leqslant & \limsup _{t \rightarrow+\infty} \frac{t_{\delta} C_{\delta}}{t}+\limsup _{t \rightarrow+\infty} \frac{\left(t-t_{\delta}\right) \epsilon}{t} \leqslant \epsilon .
\end{aligned}
$$

By the arbitrariness of $\epsilon>0$, we obtain the desired assertion that the positive linear functional $\mathcal{L}_{u}(\psi)$ depends only on the values of $\psi$ on the trajectory attractor $\mathcal{A}_{H}^{\text {tr }}$.

We now define $G(\psi)=\mathcal{L}_{u}(\ell(\psi))$ for $\psi \in \mathcal{C}\left(\mathcal{A}_{H}^{\text {tr }}\right)$, where $\ell(\psi)$ is an extension of $\psi$ from $\mathcal{C}\left(\mathcal{A}_{H}^{\text {tr }}\right)$ to $\mathcal{C}\left(\mathcal{F}^{+}\right)$given by the Tietze theorem (see [12, Theorem A.7]). By Definition 4.2(1) and (4.7), we can prove that $G(\cdot)$ is a positive linear functional on $\mathcal{C}\left(\mathcal{A}_{H}^{\mathrm{tr}}\right)$. Since $\mathcal{A}_{H}^{\mathrm{tr}} \subset \mathcal{T}_{H}^{+}$, and $\mathcal{T}_{H}^{+}$is a locally compact topological space, we see, by the Kakutani-Riesz Representation Theorem (see [12, Theorem A.1]), that there exists a unique positive, finite, Borel measure $\rho_{u}$ on $\mathcal{A}_{H}^{\text {tr }}$ such that

$$
G(\psi)=\int_{\mathcal{A}_{H}^{\mathrm{tr}}} \psi(v) \mathrm{d} \rho_{u}(v) .
$$

We extend $\rho_{u}$ (by zero) to a Borel measure on $\mathcal{F}^{+}$by taking $\rho_{u}(E)=\rho_{u}\left(\mathcal{A}_{H}^{\text {tr }} \cap E\right)$ for $E \in \mathcal{B}\left(\mathcal{F}^{+}\right)$. So for every $\psi \in \mathcal{C}\left(\mathcal{F}^{+}\right)$,

$$
G(\psi)=\mathcal{L}_{u}(\psi)=\operatorname{LIM}_{t \rightarrow+\infty} \frac{1}{t} \int_{0}^{t} \psi(S(s) u) \mathrm{d} s=\int_{\mathcal{A}_{H}^{\text {tr }}} \psi(v) \mathrm{d} \rho_{u}(v)=\int_{\mathcal{F}^{+}} \psi(v) \mathrm{d} \rho_{u}(v),
$$

and clearly $\rho_{u}\left(\mathcal{F}^{+} \backslash \mathcal{A}_{H}^{\text {tr }}\right)=0$. At the same time, since $\mathcal{F}^{+}$is a metrizable space (cf. [13, Proposition 2.1(2)]), every finite Borel measure is tight in the sense of Definition 4.1(1) (see [1, Theorem 12.5]. Therefore, $\rho_{u}$ is a trajectory statistical solution for the 3D globally modified Navier-Stokes equations. 
We next prove that $\rho_{u}$ is invariant under the action of $\{S(t)\}_{t \geqslant 0}$. To this end, we fix any $t^{*} \geqslant 0$ and any $\psi \in \mathcal{C}\left(\mathcal{F}^{+}\right)$. Since the interval $\left[0, t^{*}\right]$ is compact and $s \mapsto|\psi(S(s) u)|$ is continuous, we have

$$
\operatorname{LIM}_{t \rightarrow+\infty} \frac{1}{t} \int_{0}^{t^{*}} \psi(S(s) u) \mathrm{d} s=0
$$

At the same time, we use (4.1) and (4.5) to get

$$
\left|\operatorname{LIM}_{t \rightarrow+\infty} \frac{1}{t} \int_{t}^{t+t^{*}} \psi(S(s) u) \mathrm{d} s\right| \leqslant \limsup _{t \rightarrow+\infty} \frac{t^{*}}{t} C_{1}=0 .
$$

Therefore, (4.10) and (4.11) give

$$
\begin{aligned}
\int_{\mathcal{F}^{+}} \psi\left(S\left(t^{*}\right) v\right) \mathrm{d} \rho_{u}(v)= & \operatorname{LIM}_{t \rightarrow+\infty} \frac{1}{t} \int_{0}^{t} \psi\left(S\left(t^{*}\right) S(s) u\right) \mathrm{d} s \\
= & \operatorname{LIM}_{t \rightarrow+\infty} \frac{1}{t} \int_{0}^{t} \psi\left(S\left(t^{*}+s\right) u\right) \mathrm{d} s \\
= & \operatorname{LIM}_{t \rightarrow+\infty} \frac{1}{t} \int_{t^{*}}^{t+t *} \psi(S(s) u) \mathrm{d} s \\
= & \operatorname{LIM}_{t \rightarrow+\infty} \frac{1}{t} \int_{t}^{t+t^{*}} \psi(S(s) u) \mathrm{d} s-\operatorname{LIM}_{t \rightarrow+\infty} \frac{1}{t} \int_{0}^{t^{*}} \psi(S(s) u) \mathrm{d} s \\
& +\operatorname{LIM}_{t \rightarrow+\infty} \frac{1}{t} \int_{0}^{t} \psi(S(s) u) \mathrm{d} s \\
= & \operatorname{LIM}_{t \rightarrow+\infty} \frac{1}{t} \int_{0}^{t} \psi(S(s) u) \mathrm{d} s=\int_{\mathcal{F}^{+}} \psi(v) \mathrm{d} \rho_{u}(v),
\end{aligned}
$$

which is the desired invariant property. Finally, remember that the regularity of the trajectory statistical solution means that it is supported by a set in the trajectory space in which all weak solutions are in fact strong solutions. This is a directly conclusion of the regularity of the trajectory attractor which has been proved in Theorem 3.1(3). The proof is completed.

\section{$5 \quad$ Summary and Remark}

In this article, we first prove the existence and regularity of the trajectory attractor for a 3D system of globally modified Navier-Stokes equations. The regularity of the trajectory attractor reveals the trajectory asymptotic smoothing effect of the addressed equations in the sense that the trajectories become eventually more smoother than the initial values. We then use the natural translation semigroup and trajectory attractor to construct trajectory statistical solution for the 3D globally modified Navier-Stokes equations. The constructed trajectory statistical solution is a tight Borel probability measure, which is supported by the trajectory attractor and is invariant under the action of the translation semigroup. Moreover, the constructed trajectory statistical solution is asymptotic regular in the sense that it is supported by a set in the trajectory space in which all weak solutions are in fact strong solutions. 
We believe that the approach using trajectory attractor to construct trajectory statistical solution here can be raised to an abstract level for a broad class of evolutionary equations displaying the property of global existence of weak solutions without a known result of global uniqueness, such as the 2D dissipative Euler equations [9], the 3D MHD equations $[7,14,32,38]$ and the 3D convective Brinkman-Forchheimer equations [36]. The fundamental hypotheses on the concrete evolutionary equations is that the trajectory space is a metrizable normal topological space and the natural translation semigroup possesses a compact trajectory attractor.

We end this article with a remark. If we set $\mu_{t}=S(t) \rho_{u}$ (where $\rho_{u}$ is the Borel probability measure obtained in Theorem 4.1) and rewrite equation (2.4) as

$$
\frac{\mathrm{d} u}{\mathrm{~d} t}=\mathcal{G}(u)
$$

for $\mathcal{G}(u)=g-A u+B_{N}(u, u)$, then we can prove the following Liouville-type equation in Statistical Mechanics

$$
\frac{\mathrm{d}}{\mathrm{d} t} \int_{\mathcal{F}^{+}} \Phi(v) \mathrm{d} \mu_{t}(v)=\int_{\mathcal{F}^{+}}\left(\mathcal{G}(v), \Phi^{\prime}(v)\right) \mathrm{d} \mu_{t}(v)
$$

for all "test" functions $\Phi$ (cf. [12, $\mathrm{P}_{178}$, Definition 1.2]). We will investigate this issue in another paper.

\section{References}

[1] C. D. Aliprentis, K. C. Border, Infinite Dimensional Analysis, A Hithhiker's Guide, third editon, Springer-Verlag, 2006.

[2] A. Bronzi, C. F. Mondaini, R. Rosa, Abstract framework for the theory of statistical solutions, J. Differential Equations, 260(2016), 8428-8484.

[3] T. Caraballo, J. Real, P. E. Kloeden, Unique strong solutions and $V$-attractor of a three dimensional system of globally modified Navier-Stokes equations, $A d v$. Nonlinear Studies, 6(2006), 411-436.

[4] T. Caraballo, G. Łukaszewicz, J. Real, Invariant measures and statitical solutions of the globally modified Navier-Stokes equations, Discrete Cont. Dyn. Syst.-B, 10(2008), 761-781.

[5] T. Caraballo, J. Real, P. E. Kloeden, Unique strong solutions and $V$-Attractors of a three dimensional system of globally modified Navier-Stokes equations, $A d v$. Nonlinear Studies, 10(2010), 245-247.

[6] M. Chekroun, N. E. Glatt-Holtz, Invariant measures for dissipative dynamical systems: Abstract results and applications, Comm. Math. Phys., 316(2012), 723-761.

[7] C. Cao, J, Wu, Two regularity criteria for the 3D MHD equations, J. Differential Equations, 248(2010), 2263-2274.

[8] V. V. Chepyzhov, M. I. Vishik, Attractors for Equations of Mathematical Physics. American Mathematical Society Colloquium Publications, 49, Providence, R.I., 2002. 
[9] V. V. Chepyzhov, M. I. Vishik, S. V. Zelik, Strong trajectory attractor for dissipative Euler equations, J. Math. Pures Appl., 96(2011), 395-407.

[10] B. Dong, J. Song, Globally regularity and asymptotic behavior of modified NavierStokes equations with fractional dissipation, Discrete Cont. Dyn. Syst.-A, 32(2012), 57-79.

[11] C. Foias, G. Prodi, Sur les solutions statistiques equations de Navier-Stokes, Ann. Math. Pura Appl., 111(1976), 307-330.

[12] C. Foias, O. Manley, R. Rosa, R. Temam, Navier-Stokes Equations and Turbulence, Cambridge University Press, Cambridge, 2001.

[13] C. Foias, R. Rosa, R. Temam, Properties of time-dependent statistical solutions of the three-dimensional Navier-Stokes equations, Annales de L'Institut Fourier, 63(2013), 2515-2573.

[14] X. Jia, Y. Zhou, On regularity criteria for the 3D incompressible MHD equations involving one velocity component, J. Math. Fluid Mech., 18(2016), 187-206.

[15] P. E. Kloeden, J. A. Langa, J. Real, Pullback $V$-attractors of the three dimensional system of nonautonomous globally modified Navier-Stokes equations: existence and finite fractal dimension, Comm. Pure Appl. Anal., 6(2007), 937-955.

[16] P. E. Kloeden, J. Valero, The weak connecttedness of the attainability set of weak solutions of the 3D Navier-Stokes equations, Proc. Roy. Soc. London A, 463(2007), 1491-1508.

[17] P. E. Kloeden, P. Marín-Rubio, J. Real, Equivalence of invariant measures and stationary statistical solutions for the autonomous globally modified Navier-Stokes equations, Comm. Pure Appl. Anal., 8(2009), 785-802.

[18] O. A. Ladyzhenskaya, The Mathematical Theory of Viscous Incompressible Flow, Gordon and Breach, New York, 1969.

[19] X. Li, W. Shen, C. Sun, Invariant measures for complex-valued dissipative dynamical systems and applications, Discrete Cont. Dyn. Syst.-B, 22(2017), 2427-2446.

[20] G. Łukaszewicz, Pullback attractors and statistical solutions for 2-D Navier-Stokes equations, Discrete Cont. Dyn. Syst.-B, 9(2008), 643-659.

[21] G. Łukaszewicz, J. Real, J. C. Robinson, Invariant measures for dissipative dynamical systems and generalised Banach limits, J. Dynam. Differential Equations, 23(2011), 225-250.

[22] G. Łukaszewicz, J. C. Robinson, Invariant measures for non-autonomous dissipative dynamical systems, Discrete Cont. Dyn. Syst.-A, 34(2014), 4211-4222.

[23] P. Marín-Rubio, A. M. Márquez-Durán, J. Real, Three-dimensional system of globally modified Navier-Stokes equations with infinite delays, Discrete Cont. Dyn. Syst.-B, 14(2010), 655-673.

[24] P. Marín-Rubio, A. M. Márquez-Durán, J. Real, Pullback attractors for globally modified Navier-Stokes equations with infinite delays, Discrete Cont. Dyn. Syst.-A, 31(2011), 779-796. 
[25] P. Marín-Rubio, A. M. Márquez-Durán, J. Real, Asymptotic behavior of solutions for a three dimensional system of globally modified Navier-Stokes equations with a locally Lipschitz delay term, Nonlinear Anal., 79(2013), 68-79.

[26] Cláudia B. Gentile Moussa, Invariant measures for multivalued semigroups, $J$. Math. Anal. Appl., 455(2017), 1234-1248.

[27] M. Romito, The uniqueness of weak solutions of the globally modified NavierStokes equations, Adv. Nonlinear Studies, 9(2009), 425-427.

[28] R. Temam, Navier-Stokes Equations (Theory and Numerical Analysis), NorthHolland, Amsterdam, 1984.

[29] M. I. Vishik, A. V. Fursikov, Translationally homogeous statistical solutions and individual solutions with infinite energy of a system of Navier-Stokes equations, Siberian Mathematical Journal, 19(1978), 710-729.

[30] M. I. Vishik, V. V. Chepyzhov, Trajectory and global attractors of threedimensional Navier-Stokes systems, Math. Notes, 77(2002), 177-193.

[31] M. I. Vishik, V. V. Chepzhov, Trajectory attractors of equations of mathematical physics, Russian Math. Surveys, 4(2011), 639-731.

[32] J. Wu, Regularity results for weak solutions of the 3D MHD equations, Discrete Cont. Dyn. Syst.-A, 10(2004), 543-556.

[33] X. Wang, Upper-semicontinuity of stationary statistical properties of dissipative systems, Discrete Cont. Dyn. Syst.-A, 23(2009), 521-540.

[34] C. Zhao, S. Zhou, Y. Li, Trajectory attractor and global attractor for a twodimensional incompressible non-Newtonian fluid, J. Math. Anal. Appl., 325(2007), 1350-1362.

[35] C. Zhao, Y. Li, S. Zhou, Regularity of trajectory attractor and upper semicontinuity of global attractor for a 2D non-Newtonian fluid, J. Differential Equations, 247(2009), 2331-2363.

[36] C. Zhao, L. Kong, G. Liu, M. Zhao, The trajectory attractor and its limiting behavior for the convective Brinkman-Forchheimer equations, Topological Meth. Nonl. Anal., 44(2014), 413-433.

[37] C. Zhao, L. Yang, Pullback attractor and invariant measure for the globally modified Navier-Stokes equations, Comm. Math. Sci., 15(2017), 1565-1580.

[38] Y. Zhou, S. Gala, Regularity criteria for the solutions to the 3D MHD equations in the multiplier space, Z. Angew. Math. Phys., 61 (2010), 193-199. 